ESAIM: M2AN 50 (2016) 1731-1761

DOI: $10.1051 / \mathrm{m} 2 \mathrm{an} / 2016002$
ESAIM: Mathematical Modelling and Numerical Analysis

www.esaim-m2an.org

\title{
DETECTABILITY AND STATE ESTIMATION FOR LINEAR AGE-STRUCTURED POPULATION DIFFUSION MODELS
}

\author{
Karim RAmDani ${ }^{1}$, Marius Tucsnak ${ }^{2}$ And Julie VALEIN $^{2}$
}

\begin{abstract}
We investigate a state estimation problem for an infinite dimensional system appearing in population dynamics. More precisely, given a linear model for age-structured populations with spatial diffusion, we assume the initial distribution to be unknown and that we have at our disposal an observation locally distributed in both age and space. Using Luenberger observers, we solve the inverse problem of recovering asymptotically in time the distribution of population. The observer is designed using a finite dimensional stabilizing output injection operator, yielding an effective reconstruction method. Numerical experiments are provided showing the feasibility of the proposed reconstruction method.
\end{abstract}

Mathematics Subject Classification. 92D25, 35R30, 93D15, 93B55.

Received April 13, 2015. Revised January 4, 2016. Accepted January 6, 2016.

\section{INTRODUCTION AND PROBLEM SETTING}

We consider a linear age-structured population model with spatial diffusion described by the following system:

$$
\left\{\begin{aligned}
\partial_{t} p(a, x, t) & +\partial_{a} p(a, x, t), & & a \in\left(0, a^{*}\right), x \in \Omega, t>0, \\
& =-\mu(a) p(a, x, t)+k \Delta p(a, x, t), & & \\
p(a, x, t) & =0, & & a \in\left(0, a^{*}\right), x \in \partial \Omega, t>0, \\
p(a, x, 0) & =p_{0}(a, x), & & a \in\left(0, a^{*}\right), x \in \Omega, \\
p(0, x, t) & =\int_{0}^{a^{*}} \beta(a) p(a, x, t) \mathrm{d} a, & & x \in \Omega, t>0 .
\end{aligned}\right.
$$

In the above equations:

- $\Omega \subset \mathbb{R}^{n}, n \geqslant 1$, denotes a smooth bounded domain, $k$ is a positive constant diffusion coefficient and $\Delta$ the laplacian with respect to the space variable $x$.

- $p(a, x, t)$ denotes the distribution density of the population of age $a$ at spatial position $x \in \Omega$ at time $t$;

\footnotetext{
Keywords and phrases. Inverse problems, observers, stabilization, population dynamics, spatial diffusion.

1 Inria, 54600 Villers-lès-Nancy, France. karim.ramdani@inria.fr

2 Université de Lorraine, Institut Élie Cartan de Lorraine, UMR 7502, 54506 Vandœuvre-lès-Nancy, France.
} 


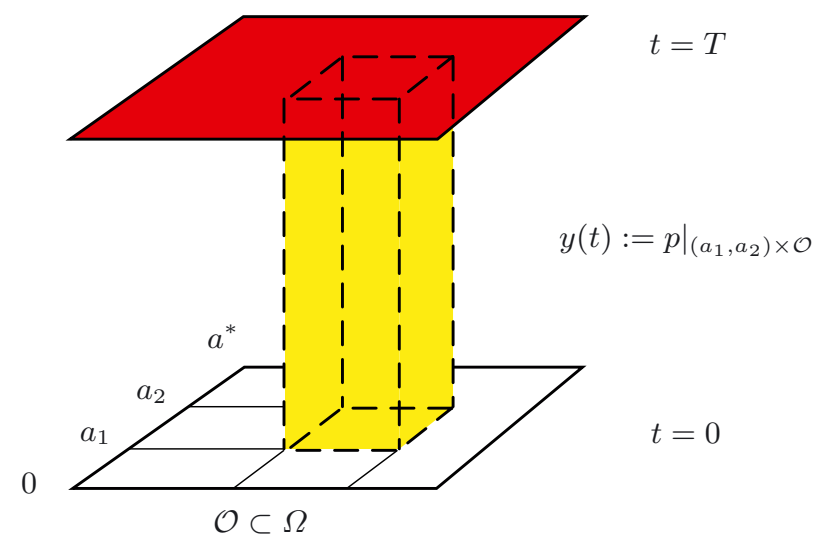

Figure 1 . How to estimate $p(T)$ as $T \rightarrow+\infty$ from the knowledge of $y(t)$ for $t \in(0, T)$ ?

- $p_{0}$ denotes the initial distribution;

- $a^{*} \in(0,+\infty)$ is the maximal life expectancy;

- $\beta(a)$ and $\mu(a)$ are positive functions denoting respectively the birth and death rates, which are supposed to be independent of $x$ (see Fig. 5 for typical graphs of these functions).

The last equation in (1.1) describing the birth process is the so-called renewal equation. We assume here homogeneous Dirichlet boundary conditions (in space) which model a hostile habitat at the boundary $\partial \Omega$, but other type of boundary conditions (in space) can also be considered (typically Neumann homogeneous boundary conditions which correspond to isolated population, i.e. vanishing incoming and outgoing flux).

In this paper, we investigate the following inverse problem (see Fig. 1):

Assuming the initial age distribution $p_{0}$ to be unknown, but knowing the age distribution

$$
y(a, x, t):=p(a, x, t), \quad t \in(0, T), a \in\left(a_{1}, a_{2}\right), x \in \mathcal{O},
$$

where $\mathcal{O}$ is some given subset of $\Omega$ and $0 \leqslant a_{1}<a_{2} \leqslant a$, is it possible, and if so how, to estimate when $T \rightarrow+\infty$ the age distribution $p(a, x, T)$, for $x \in \Omega$ and $a \in\left(0, a^{*}\right)$ ?

We provide a positive answer to this question and we propose an effective reconstruction algorithm. Based on a semigroup formulation, our method crucially uses two main ingredients: the Luenberger observers [44] and the fact that the problem has finitely many unstable eigenvalues (i.e. eigenvalues $\lambda$ such that $\operatorname{Re} \lambda \geqslant 0$ ). Let us make this statement more precise by outlining in a formal way the main ideas of our reconstruction method.

Using a semigroup formulation, we first rewrite problem (1.1) in the abstract form (throughout the paper, the dot denotes the derivative with respect to time)

$$
\left\{\begin{array}{l}
\dot{p}(t)=A p(t), \quad t \in(0, T) \\
p(0)=p_{0}
\end{array}\right.
$$

where $A: \mathcal{D}(A) \rightarrow X$ is the generator of a $C_{0}$-semigroup on a Hilbert space $X$. Similarly, the available observation can also be reformulated using a bounded observation operator $C \in \mathcal{L}(X, Y)$, where $Y$ is another Hilbert space:

$$
y(t)=C p(t), \quad t \in(0, T) .
$$


We define the Luenberger type observer as the solution $\widehat{p}$ of the following system

$$
\left\{\begin{array}{l}
\dot{\hat{p}}(t)=A \widehat{p}(t)+L(C \widehat{p}(t)-y(t)), \quad t \in(0, T) \\
\widehat{p}(0)=0
\end{array}\right.
$$

where $L \in \mathcal{L}(Y, X)$ is a linear operator to be chosen in such way that $A+L C$ generates an exponentially stable semigroup on $X$. Indeed, since the error $e:=\widehat{p}-p$ satisfies by construction

$$
e(t)=(A+L C) e(t), \quad t \in(0, T)
$$

this condition ensures that $e(T)$ converges exponentially to 0 , i.e. that $(\|\cdot\|$ denotes the norm on $X)$

$$
\|\widehat{p}(T)-p(T)\| \leqslant M \mathrm{e}^{-\omega T}\left\|p_{0}\right\|
$$

showing that $\widehat{p}(T)$ constitutes an approximation of $p(T)$ as $T \rightarrow+\infty$. Let us emphasize that $\widehat{p}(T)$ can be computed (by solving (1.2)) exclusively from the knowledge of the observation $y(t)$, provided we have determined a stabilizing output injection operator $L$.

To compute this operator, we use the second main ingredient of our method: the fact that $A$ has finitely many unstable eigenvalues. Following Triggiani [63,64], we will show that the operator $L$ can be easily deduced from the finite dimensional operator $L^{+}$stabilizing the finite dimensional system $\left(A^{+}, C^{+}\right)$, where $A^{+}$is the restriction of $A$ to the subspace $X^{+}$of $X$ spanned by the unstable (generalized) eigenfunctions of $A$ and $C^{+}$is the corresponding observation operator.

For an overview on population dynamics models, we refer the reader to the monograph of Webb [67] and to the introductory paper [68]. Gurtin [27] introduced the first model with spatial diffusion (see also Gurtin and MacCamy [28]). For linear models, existence of a semigroup in $L^{2}$ and large time asymptotics have been developed by Chan and Guo in the case of homogeneous Dirichlet boundary (in [13] for age dependent birth and death rates and in [26] for age and space dependent birth and death rates) and by Huyer [33,34] in the case of homogeneous Neumann boundary conditions using perturbation techniques. The $L^{1}$ setting has been investigated by Rhandi [56] for a linear age-dependent model with spatial diffusion involving age and space dependent birth and death rates. More recently, Walker [66] proposed an abstract functional analytic framework to study the large time behavior for an age-structured linear diffusive model. Existence and large time behavior for non linear models have been studied by Langlais [41,42]. Exact and approximate controllability issues for age-structured dynamic systems have been studied in Ainseba and Langlais [3], Ainseba and Iannelli [1,2], Traore [60], Traore and Kavian [37].

The literature on inverse problems for population dynamics models is less rich, especially when spatial diffusion is assumed. Without spatial diffusion, Pilant and Rundel [51], Rundell [57], Engl, Rundell and Scherzer [23] studied the determination of the death rate, Gyllenberg, Osipov and Päivärinta [29] investigated uniqueness for the reconstruction of birth and death rates. State estimation problems, like the one considered here, have been considered only recently. Di Blasio and Lorenzi [21,22] studied the well-posedness of the inverse problem of reconstructing the initial state for a linear age-dependent model. Traore investigated estimation problems for population dynamics, in which the state has to be recovered from distributed observation [62] or boundary observation [61] in space and full observation in age. However, he followed a non standard data assimilation method introduced by Puel $[52,53]$ which is completely different from ours. In this approach, one computes the final state (or more precisely its coordinates in a suitably chosen Hilbert basis) by combining a zero controllability and optimal control results. To conclude, let us also mention the work of Perasso [49] in which identifiability issues are considered for an epidemiological model. 
Unless otherwise stated, we will make the following classical assumptions on $\beta$ and $\mu$ (see, for instance, $[13,20,25])$ :

- $\left(\mathbf{H}_{\beta}\right) \quad \beta \in L^{\infty}\left(0, a^{*}\right), \beta \geqslant 0$ a.e. in $\left(0, a^{*}\right)$;

- $\left(\mathbf{H}_{\mu}\right) \quad \mu \in L_{\mathrm{loc}}^{1}\left(0, a^{*}\right), \mu \geqslant 0$ a.e. in $\left(0, a^{*}\right)$ and

$$
\lim _{a \rightarrow a^{*}} \int_{0}^{a^{*}} \mu(s) \mathrm{d} s=+\infty .
$$

We also introduce the function

$$
\Pi(a):=\exp \left(-\int_{0}^{a^{*}} \mu(s) \mathrm{d} s\right)
$$

which represents the probability to survive at age $a$ (also known as the life table function). Hence, it is a decreasing function satisfying (thanks to condition (1.3))

$$
\lim _{a \rightarrow a^{*}} \Pi(a)=0 .
$$

The paper is organized as follows. Section 2 gathers some well-known facts about the population semigroup associated to (1.1) and the spectral properties of its generator $A$. In particular, we will see that $A$ has finitely many unstable eigenvalues. In Section 3, we provide some abstract results about the detectability (see Def. 3.1) of infinite dimensional linear systems $(A, C)$ where $A: \mathcal{D}(A) \rightarrow X$ is the generator of a $C_{0}$-semigroup on a Hilbert space $X$ having finitely many unstable eigenvalues, and $C \in \mathcal{L}(X, Y)$ is a bounded observation operator. In particular, we show the existence of a finite dimensional stabilizing output injection operator for such systems, provided $(A, C)$ satisfy a Hautus test (this follows from combining Thm. 3.4 and Prop. 3.5). In Section 4, we show that problem (1.1) fits into the general framework described in Section 3. This allows us to design a Luenberger type observer for (1.1) providing a solution to our estimation inverse problem. The last two sections of the paper are devoted to the implementation of our reconstruction method. In Section 5 we present finite difference full discretizations of the open loop system (used for synthetic data generation) and the observer. In Section 6, we provide numerical experiments illustrating the theoretical results and showing the efficiency of the reconstruction method.

\section{Some BACKGROUND ON THE POPULATION SEMIGROUP}

We collect below some of the existing results on the population semigroup for the linear age-structured model without (Sect. 2.1) and with (Sect. 2.2) spatial diffusion. In particular, we will recall the spectrum's structure of the semigroup generators in these two cases.

\subsection{The diffusion free population dynamics}

The diffusion free case is described by the so-called McKendrick-Von Foerster model (see [59] for the proofs of the results given below):

$$
\begin{cases}\partial_{t} p(a, t)+\partial_{a} p(a, t)=-\mu(a) p(a, t), & a \in\left(0, a^{*}\right), t>0, \\ p(a, 0)=p_{0}(a), & a \in\left(0, a^{*}\right), \\ p(0, t)=\int_{0}^{a^{*}} \beta(a) p(a, t) \mathrm{d} a, & t>0 .\end{cases}
$$

The population operator $A_{0}$ corresponding to the above system is defined as follows

$$
\begin{aligned}
& \mathcal{D}\left(A_{0}\right)=\left\{\varphi \in L^{2}\left(0, a^{*}\right) \mid \varphi(0)=\int_{0}^{a^{*}} \beta(a) \varphi(a) \mathrm{d} a ;-\frac{\mathrm{d} \varphi}{\mathrm{d} a}-\mu \varphi \in L^{2}\left(0, a^{*}\right)\right\} \\
& A_{0} \varphi=-\frac{\mathrm{d} \varphi}{\mathrm{d} a}-\mu \varphi, \quad \forall \varphi \in \mathcal{D}\left(A_{0}\right) .
\end{aligned}
$$


Theorem 2.1. The operator $A_{0}$ defined by (2.1) has compact resolvent and its spectrum is constituted of a countable (infinite) set of isolated eigenvalues with finite algebraic multiplicity. The eigenvalues $\left(\lambda_{n}^{0}\right)_{n} \geqslant 1$ of $A_{0}$ (counted without multiplicity) are the solutions of the characteristic equation

$$
F(\lambda):=\int_{0}^{a^{*}} \beta(a) \mathrm{e}^{-\lambda a} \Pi(a) \mathrm{d} a=1 .
$$

The eigenvalues $\left(\lambda_{n}^{0}\right)_{n \geqslant 1}$ are of geometric multiplicity one, the eigenspace associated to $\lambda_{n}^{0}$ being the onedimensional subspace of $L^{2}\left(0, a^{*}\right)$ generated by the function

$$
\varphi_{n}^{0}(a)=\mathrm{e}^{-\lambda_{n}^{0} a} \Pi(a)=\mathrm{e}^{-\lambda_{n}^{0} a-\int_{0}^{a^{*}} \mu(s) \mathrm{d} s} .
$$

Finally, every vertical strip of the complex plane $\alpha_{1} \leqslant \operatorname{Re}(z) \leqslant \alpha_{2}, \alpha_{1}, \alpha_{2} \in \mathbb{R}$, contains a finite number of eigenvalues of $A_{0}$.

Theorem 2.2. The operator $A_{0}$ defined by (2.1) has a unique real eigenvalue $\lambda_{1}^{0}$. Moreover, we have the following properties

(1) $\lambda_{1}^{0}$ is of algebraic multiplicity one;

(2) $\lambda_{1}^{0}>0$ (resp. $\left.\lambda_{1}^{0}<0\right)$ if and only if $F(0)>1$ (resp. $\left.F(0)<1\right)$;

(3) $\lambda_{1}^{0}$ is a real dominant eigenvalue:

$$
\lambda_{1}^{0}>\operatorname{Re}\left(\lambda_{n}^{0}\right), \quad \forall n \geqslant 2 .
$$

\subsection{The population dynamics with diffusion}

Chan and Guo [13] showed the existence of a semigroup on $L^{2}\left(\left(0, a^{*}\right) \times \Omega\right)$ for linear age-structured population model with (constant) diffusion coefficient and age dependent birth and death rates in [13]. They extended this result to the case of age and space dependent birth and death rates. For reader's convenience, we sketch their proof here in this last case (which is more general than the one under consideration in this paper).

Let $X:=L^{2}\left(\left(0, a^{*}\right) \times \Omega\right)$ and define the following unbounded operator $A$ on $X$ :

$$
\begin{gathered}
\mathcal{D}(A)=\left\{\varphi \in X \mid \varphi \in C\left(\left[0, a^{*}\right] ; L^{2}(\Omega)\right) \cap L^{2}\left(\left(\left[0, a^{*}\right] ; H_{0}^{1}(\Omega)\right)\right.\right. \\
\beta \varphi \in L^{1}\left(\left[0, a^{*}\right] ; L^{2}(\Omega)\right) ;-\partial_{a} \varphi-\mu \varphi+k \Delta \varphi \in X ; \\
\left.\varphi(0, x)=\int_{0}^{a^{*}} \beta(a, x) \varphi(a, x) \mathrm{d} a \text { for almost all } x \in \Omega\right\} \\
A \varphi=-\partial_{a} \varphi-\mu \varphi+k \Delta \varphi, \quad \forall \varphi \in \mathcal{D}(A) .
\end{gathered}
$$

Assume that $\beta$ and $\mu$ satisfy the following assumptions

- $\beta \in L^{\infty}\left(\left[0, a^{*}\right] \times \Omega\right), \beta \geqslant 0$ a.e. in $\left(0, a^{*}\right) \times \Omega$ and

$$
\text { meas }\left\{a \in\left[0, a^{*}\right] \quad \mid \inf _{x \in \bar{\Omega}} \beta(a, x)>0\right\}>0 ;
$$

- $\mu$ is measurable and non negative on $[0, a] \times \bar{\Omega}$ for every $a \in\left(0, a^{*}\right)$;

- the function $a_{0} \mapsto \sup _{a \in\left[0, a_{0}\right]} \int_{\Omega} \mu^{2}(a, x) \mathrm{d} x$ is continuous with respect to $a \in\left[0, a^{*}\right)$;

- the functions $\underline{\mu}(s)=\inf _{x \in \bar{\Omega}} \mu(s, x)$ and $\bar{\mu}(s)=\sup _{x \in \bar{\Omega}} \mu(s, x)$ satisfy

$$
\lim _{a \rightarrow a^{*}} \int_{0}^{a} \underline{\mu}(s) \mathrm{d} s=+\infty, \quad \bar{\mu} \in L^{1}[0, a], \quad a \in\left[0, a^{*}\right) .
$$


Proposition 2.3. A generates a $C^{0}$ semigroup on $X$.

Proof. We just check that $\lambda I-A$ is onto for $\lambda$ large enough. First, let us recall that from standard regularity for parabolic equations, given $s \geqslant 0$ and $\psi \in L^{2}(\Omega)$, the initial and boundary value problem of unknown $v(a, x)$ :

$$
\begin{cases}\partial_{a} v(a, x)=k \Delta v(a, x)-\mu(a, x) v(a, x), & s \leqslant a<a^{*}, x \in \Omega, \\ v(a, x)=0, & s \leqslant a<a^{*}, x \in \partial \Omega, \\ v(s, x)=\psi(x), & x \in \Omega,\end{cases}
$$

admits a unique solution

$$
v \in C\left(\left[s, a^{*}\right] ; L^{2}(\Omega)\right) \cap L^{2}\left(\left[s, a^{*}\right] ; H_{0}^{1}(\Omega)\right) .
$$

In the sequel, we denote the solution operator by $\mathcal{F}(a, s)$, in the sense that

$$
v(a, x)=\mathcal{F}(a, s) \psi(x), \quad 0 \leqslant s \leqslant a \leqslant a^{*}, x \in \Omega .
$$

Now, given $\lambda \in \mathbb{R}, f \in X$ and $\psi \in L^{2}(\Omega)$, the initial and boundary value problem

$$
\begin{cases}\partial_{a} v(a, x)=k \Delta v(a, x)-(\mu(a, x)+\lambda) v(a, x)+f(a, x), & 0 \leqslant a<a^{*}, x \in \Omega, \\ v(a, x)=0, & 0 \leqslant a<a^{*}, x \in \partial \Omega, \\ v(0, x)=\psi(x), & x \in \Omega,\end{cases}
$$

admits a unique solution

$$
v \in C\left(\left[0, a^{*}\right] ; L^{2}(\Omega)\right) \cap L^{2}\left(\left[0, a^{*}\right] ; H_{0}^{1}(\Omega)\right),
$$

given by

$$
v(a, \cdot)=\mathrm{e}^{-\lambda a} \mathcal{F}(a, 0) \psi+\int_{0}^{a} \mathrm{e}^{-\lambda(a-s)} \mathcal{F}(a, s) f(s, \cdot) \mathrm{d} s \quad\left(0 \leqslant a<a^{*}\right) .
$$

It follows that

$$
\begin{aligned}
v(0, x)-\int_{0}^{a^{*}} \beta(a, x) v(a, x) \mathrm{d} a= & \psi(x)-\int_{0}^{a^{*}} \mathrm{e}^{-\lambda a} \beta(a, x) \mathcal{F}(a, 0) \psi(x) \mathrm{d} a \\
& -\int_{0}^{a^{*}} \beta(a, x) \int_{0}^{a} \mathrm{e}^{-\lambda(a-s)} \mathcal{F}(a, s) f(s, x) \mathrm{d} s \mathrm{~d} a .
\end{aligned}
$$

On the other hand, consider the operator $\mathcal{B}_{\lambda}$ defined by

$$
\left(\mathcal{B}_{\lambda} \psi\right)(x)=\int_{0}^{a^{*}} \mathrm{e}^{-\lambda a} \beta(a, x) \mathcal{F}(a, 0) \psi(x) \mathrm{d} a \quad\left(\psi \in L^{2}(\Omega)\right) .
$$

We clearly have that $\mathcal{B}_{\lambda} \in \mathcal{L}\left(L^{2}(\Omega)\right)$ for every $\lambda \in \mathbb{R}$ and that $\lim _{\lambda \rightarrow \infty}\left\|\mathcal{B}_{\lambda}\right\|_{\mathcal{L}\left(L^{2}(\Omega)\right)}=0$. This implies that $I-\mathcal{B}_{\lambda}$ is invertible for $\lambda$ large enough. Taking

$$
\psi=\left(I-\mathcal{B}_{\lambda}\right)^{-1} \int_{0}^{a^{*}} \beta(a, \cdot) \int_{0}^{a} \mathrm{e}^{-\lambda(a-s)} \mathcal{F}(a, s) f(s, \cdot) \mathrm{d} s \mathrm{~d} a,
$$

and using (2.6), it follows that $v$ defined by (2.5) lies in $\mathcal{D}(A)$ and $\lambda v-A v=f$. We have thus shown that $A$ is a semigroup generator. 


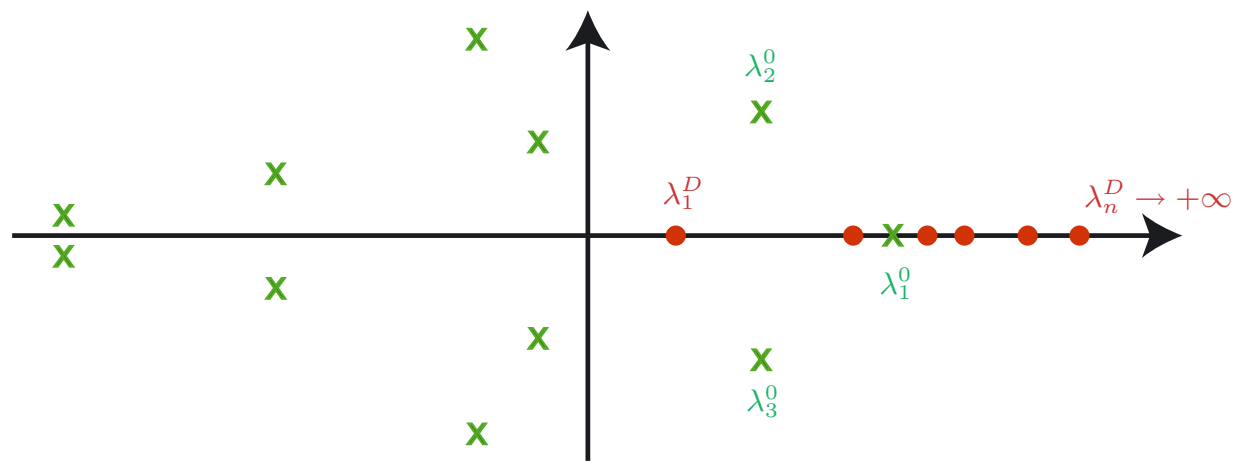

Figure 2. The spectra of the diffusion free operator $A_{0}$ (green crosses) and of $-k \Delta$ (red circles). (Color online)

Under assumptions $\left(\mathbf{H}_{\beta}\right)-\left(\mathbf{H}_{\mu}\right)$, problem (1.1) obviously fits into the above framework. Hence, defining $A$ by $(2.3)$ and (2.4), we can write problem (1.1) in the abstract form:

$$
\left\{\begin{array}{l}
\dot{p}(t):=A p(t), \quad t>0 \\
p(0)=p_{0}
\end{array}\right.
$$

The generator $A$ of the population semigroup is the sum of a population operator without diffusion $-\mathrm{d} / \mathrm{d} a-\mu I$ and a spatial diffusion term $k \Delta$. It turns out that spectral properties of $A$ can be easily obtained from those of these two operators, as it can be seen from the following result, proved by Chan and Guo [13].

Theorem 2.4. Let $0<\lambda_{1}^{D}<\lambda_{2}^{D} \leqslant \lambda_{3}^{D} \leqslant \ldots$ be the increasing sequence of eigenvalues of $-k \Delta$ with Dirichlet boundary conditions and let $\left(\varphi_{n}^{D}\right)_{n \geqslant 1}$ be a corresponding orthonormal basis of $L^{2}(\Omega)$. Let $\left(\lambda_{n}^{0}\right)_{n \geqslant 1}$ and $\left(\varphi_{n}^{0}\right)_{n \geqslant 1}$ be respectively the sequence of eigenvalues and eigenfunctions of the free diffusion operator $A_{0}$ defined by (2.1) (see Thm. 2.1). Then the following assertions hold:

(1) The operator $A$ has compact resolvent and its eigenvalues are given by

$$
\sigma(A)=\left\{\lambda_{i}^{0}-\lambda_{j}^{D} \mid i, j \in \mathbb{N}^{*}\right\} .
$$

(2) A has a real dominant eigenvalue:

$$
\lambda_{1}=\lambda_{1}^{0}-\lambda_{1}^{D}>\operatorname{Re}(\lambda), \quad \forall \lambda \in \sigma(A), \lambda \neq \lambda_{1} .
$$

Moreover, $\lambda_{1}$ is a simple eigenvalue, the corresponding eigenspace being generated by

$$
\varphi_{1}(a, x):=\varphi_{1}^{0}(a) \varphi_{1}^{D}(x)=\mathrm{e}^{-\lambda_{1}^{0} a} \Pi(a) \varphi_{1}^{D}(x) .
$$

(3) The eigenspace associated to an eigenvalue $\lambda$ of $A$ is given by

$$
\operatorname{Span}\left\{\varphi_{i}^{0}(a) \varphi_{j}^{D}(x)=\mathrm{e}^{-\lambda_{i}^{0} a} \Pi(a) \varphi_{j}^{D}(x) \mid \lambda_{i}^{0}-\lambda_{j}^{D}=\lambda\right\} .
$$

Remark 2.5. The above spectral results without and with diffusion show that every vertical strip of the complex plane contains a finite number of eigenvalues of $A$ and that the number of unstable eigenvalues is finite.

We also have the following useful result (see [13], Thm. 2):

Proposition 2.6. The semigroup $\mathrm{e}^{t A}$ generated on $X$ by $A$ is compact for $t \geqslant a^{*}$. 
According to Zabczyk [69], Section 2, this implies in particular that

$$
\omega_{a}(A)=\omega_{0}(A)
$$

where $\omega_{a}(A):=\lim _{t \rightarrow+\infty} t^{-1} \ln \left\|e^{t A}\right\|$ denotes the growth bound of the semigroup $e^{t A}$ and $\omega_{0}(A):=\sup \{\operatorname{Re} \lambda \mid$ $\lambda \in \sigma(A)\}$ the spectral bound of $A$. It is worth noticing that the above condition ensures that the exponential stability of $e^{t A}$ is equivalent to the condition $\omega_{0}(A)<0$.

\section{Detectability of infinite-Dimensional Systems With Finitely Many UnStable EIGENVALUES}

In this section, we derive some results about the detectability of infinite-dimensional systems with finitely many unstable eigenvalues, paying a special care to the diagonalizable case (Sect. 3.2). These results, which are derived in an abstract framework, will be applied in Section 4 to design an observer for (1.1).

\subsection{General case}

Let $A: \mathcal{D}(A) \rightarrow X$ be a linear operator with compact resolvents on a Hilbert space $X$ generating a $C_{0}$-semigroup in $X$, and let $C \in \mathcal{L}(X, Y)$, where $Y$ is another Hilbert space. We assume that $A$ satisfies the following assumptions

(A.1) $A$ admits $M$ eigenvalues (counted without multiplicities) with real part greater or equal than 0 . More precisely we can reorder the eigenvalues $\left(\lambda_{n}\right)_{n \in \mathbb{N}^{*}}$ of $A$ so that the sequence $\left(\operatorname{Re} \lambda_{n}\right)_{n \in \mathbb{N}^{*}}$ is nonincreasing, and we suppose that $M \in \mathbb{N}^{*}$ is such that

$$
\ldots \leqslant \operatorname{Re} \lambda_{M+1}<0 \leqslant \operatorname{Re} \lambda_{M} \leqslant \ldots \leqslant \operatorname{Re} \lambda_{2} \leqslant \operatorname{Re} \lambda_{1} .
$$

(A.2) We have the equality

$$
\omega_{a}(A)=\omega_{0}(A)
$$

where $\omega_{a}(A):=\lim _{t \rightarrow+\infty} t^{-1} \ln \left\|e^{t A}\right\|$ denotes the growth bound of $e^{t A}$ and $\omega_{0}(A):=\sup \{\operatorname{Re} \lambda \mid \lambda \in \sigma(A)\}$ the spectral bound of $A$.

Note that assumption (A.2) is in particular satisfied if $e^{t A}$ is an analytic semigroup or if $e^{t A}$ is compact for some $t$ (see [69], p. 252).

Definition 3.1. The pair $(A, C)$ is detectable if there exists $L \in \mathcal{L}(Y, X)$ such that $(A+L C)$ generates an exponentially stable semigroup. Such an operator $L$ is called a stabilizing output injection operator for $(A, C)$.

In this section, our goal is to show that the detectability of the infinite dimensional system $(A, C)$ can be deduced from the detectability of the finite dimensional system corresponding to the unstable part of $A$ (namely the projection on the finite dimensional space associated with the unstable eigenvalues $\lambda_{1}, \ldots, \lambda_{M}$ ) provided a Hautus type assumption is satisfied. The results of this section can be seen as dual to those obtained in [63] for the stabilization of infinite-dimensional systems having finitely many unstable eigenvalues. Later on, this approach has been successfully used for the control and stabilization of parabolic systems $[17,65]$, especially in fluid dynamics $[9,10,12,55]$.

We set $\Sigma_{+}:=\left\{\lambda_{1}, \ldots, \lambda_{M}\right\}$ and let $\Gamma_{+}$be a positively oriented curve enclosing $\Sigma_{+}$but no other point of the spectrum $\sigma(A)$ of $A$. Let $P_{+}: X \rightarrow X$ be the projection operator defined by

$$
P_{+}:=-\frac{1}{2 \pi i} \int_{\Gamma_{+}}(\xi-A)^{-1} \mathrm{~d} \xi
$$


We set $X_{+}:=P_{+} X$ and $X_{-}:=\left(I-P_{+}\right) X$, and then $P_{+}$provides the following decomposition of $X$

$$
X=X_{+} \oplus X_{-} .
$$

Moreover $X_{+}$and $X_{-}$are invariant subspaces under $A$ (since $A$ and $P_{+}$commute) and the spectra of the restricted operators $\left.A\right|_{X_{+}}$and $\left.A\right|_{X_{-}}$are respectively $\Sigma_{+}$and $\sigma(A) \backslash \Sigma_{+}$(see [36]). We also set

$$
A_{+}:=\left.A\right|_{\mathcal{D}(A) \cap X_{+}}: \mathcal{D}(A) \cap X_{+} \rightarrow X_{+} \quad \text { and } \quad A_{-}:=\left.A\right|_{\mathcal{D}(A) \cap X_{-}}: \mathcal{D}(A) \cap X_{-} \rightarrow X_{-} .
$$

Remark 3.2. The space $X_{+}$is the finite dimensional space spanned by the generalized eigenfunctions of $A$ associated to the unstable eigenvalues (i.e. the eigenvalues contained in $\Sigma_{+}$):

$$
X_{+}=\bigoplus_{k=1}^{M} \operatorname{Ker}\left(A-\lambda_{k}\right)^{m_{k}^{\mathrm{P}}},
$$

where $m_{k}^{\mathrm{P}}$ is the multiplicity of the pole $\lambda_{k}$ in the resolvent. If $m_{k}^{\mathrm{A}}:=\operatorname{dim} \operatorname{Ker}\left(A-\lambda_{k}\right)^{m_{k}^{\mathrm{P}}}$ denotes the algebraic multiplicity of $\lambda_{k}, k=1, \ldots, M$, then the dimension of $X_{+}$is the sum of the algebraic multiplicities:

$$
\operatorname{dim} X_{+}=\sum_{k=1}^{M} m_{k}^{\mathrm{A}} .
$$

Remark 3.3. The operator $P_{+}$is not necessarily an orthogonal projection. More precisely, if we define the projection operator corresponding to $A^{*}$ :

$$
P_{+}^{*}:=-\frac{1}{2 \pi i} \int_{\overline{\Gamma_{+}}}\left(\xi-A^{*}\right)^{-1} \mathrm{~d} \xi
$$

and if we set $X_{+}^{*}:=P_{+}^{*} X$ and $X_{-}^{*}:=\left(I-P_{+}^{*}\right) X$, then we have

$$
\left(X_{+}\right)^{\perp}=X_{-}^{*} \quad \text { and } \quad\left(X_{+}^{*}\right)^{\perp}=X_{-} .
$$

We state now the main result of this section.

Theorem 3.4. Let $Q_{+}: Y \rightarrow Y_{+}:=C X_{+}$be the orthogonal projection operator from $Y$ to $Y_{+}, i_{X_{+}}: X_{+} \rightarrow X$ be the injection operator from $X_{+}$into $X$ and let

$$
C_{+}=C i_{X_{+}} \in \mathcal{L}\left(X_{+}, Y_{+}\right) .
$$

Assume that the finite dimensional projected system $\left(A_{+}, C_{+}\right)$is detectable through a stabilizing output injection operator $L_{+} \in \mathcal{L}\left(Y_{+}, X_{+}\right)$. Then, the infinite dimensional system $(A, C)$ is detectable through the stabilizing output injection operator

$$
L=i_{X_{+}} L_{+} Q_{+} \in \mathcal{L}(Y, X)
$$

Proof. Throughout the proof, we denote by $K$ a positive constant that may change from line to line. For $L \in \mathcal{L}(Y, X)$, consider the system

$$
\dot{z}(t)=A z(t)+L C z(t) .
$$

If we write $z=z_{+}+z_{-}$where $z_{+}:=P_{+} z$ and $z_{-}:=\left(I-P_{+}\right) z$, by applying $P_{+}$and $\left(I-P_{+}\right)$to (3.6), there is a corresponding splitting of (3.6) into two equations satisfied by $z_{+}$and $z_{-}$respectively

$$
\dot{z}_{+}(t)=A_{+} z_{+}(t)+P_{+} L C z(t) \quad \text { and } \quad \dot{z}_{-}(t)=A_{-} z_{-}(t)+\left(I-P_{+}\right) L C z(t) .
$$


Using (3.5) and the facts that $P_{+} i_{X_{+}}=\operatorname{Id}_{X_{+}}$and $\left(I-P_{+}\right) i_{X_{+}}=0$, we obtain

$$
\dot{z}_{+}(t)=A_{+} z_{+}(t)+L_{+} Q_{+} C z(t) \quad \text { and } \quad \dot{z}_{-}(t)=A_{-} z_{-}(t) .
$$

It follows from assumption (A.2) that $z_{-}$is exponentially stable:

$$
\left\|z_{-}(t)\right\| \leqslant K \mathrm{e}^{-\omega_{-} t}\|z(0)\|
$$

where $\omega_{-}=-\operatorname{Re} \lambda_{M+1}>0$. On the other hand, by using (3.4) and since $i_{X_{+}} z_{+}=z_{+}$, we have

$$
\begin{aligned}
\dot{z}_{+}(t) & =A_{+} z_{+}(t)+L_{+} Q_{+} C\left(z_{+}(t)+z_{-}(t)\right) \\
& =A_{+} z_{+}(t)+L_{+} Q+C i_{X_{+}} z_{+}(t)+L_{+} Q_{+} C z_{-}(t) \\
& =\left(A_{+}+L_{+} C_{+}\right) z_{+}(t)+L_{+} Q_{+} C z_{-}(t) .
\end{aligned}
$$

Using Duhamel's formula, we get

$$
z_{+}(t)=\mathbb{T}_{t}^{+} z_{+}(0)+\int_{0}^{t} \mathbb{T}_{t-s}^{+} L_{+} Q_{+} C z_{-}(s) \mathrm{d} s,
$$

where $\mathbb{T}_{t}^{+}$is the semigroup generated by $\left(A_{+}+L_{+} C_{+}\right)$, which is exponentially stable by the detectability assumption, i.e. there exists $\omega_{+}>0$ such that

$$
\left\|\mathbb{T}_{t}^{+} x\right\| \leqslant K \mathrm{e}^{-\omega_{+} t}\|x\| \quad \forall x \in X_{+}, \forall t>0 .
$$

Combined with (3.7), this yields

$$
\left\|z_{+}(t)\right\| \leqslant K\left\{\mathrm{e}^{-\omega_{+} t}\left\|z_{+}(0)\right\|+\left\|L_{+}\right\|\|C\| \int_{0}^{t} \mathrm{e}^{-\omega_{+}(t-s)} \mathrm{e}^{-\omega_{-} s}\left\|z_{-}(0)\right\| \mathrm{d} s\right\},
$$

and consequently

$$
\left\|z_{+}(t)\right\| \leqslant K\left(\mathrm{e}^{-\omega_{+} t}+\left\|L_{+}\right\|\|C\| \frac{\mathrm{e}^{-\omega_{+} t}-\mathrm{e}^{-\omega_{-} t}}{\omega_{-}-\omega_{+}}\right)\left\|z_{0}\right\| .
$$

It is then sufficient to choose $\omega_{+}$small enough such that $0<\omega_{+}<\omega_{-}$to have the exponential decay of $t \mapsto z_{+}(t)$ :

$$
\left\|z_{+}(t)\right\| \leqslant K \mathrm{e}^{-\omega_{+} t}\left\|z_{0}\right\|, \quad t>0
$$

Relations (3.7) and (3.8) yield immediately the exponential decay of $z=z_{+}+z_{-}$.

The following result provides a Hautus type sufficient condition for the detectability of the finite dimensional projected system $\left(A_{+}, C_{+}\right)$.

Proposition 3.5. If the Hautus test

$$
\left(\varphi \in \mathcal{D}(A) \mid A \varphi=\lambda \varphi \text { for } \lambda \in \Sigma_{+} \text {and } C \varphi=0\right) \quad \Longrightarrow \quad \varphi=0
$$

is satisfied, then $\left(A_{+}, C_{+}\right)$is detectable.

Proof. Since $C_{+} z_{+}=C z_{+}$for any $z_{+} \in X_{+}$, if the Hautus criterion (3.9) is satisfied, then it is clear that the following Hautus test is also satisfied:

$$
\left(\varphi \in \mathcal{D}(A) \cap X_{+} \mid A_{+} \varphi=\lambda \varphi \text { and } C_{+} \varphi=0\right) \quad \Longrightarrow \quad \varphi=0 .
$$

As the above system is finite dimensional, $\left(A_{+}, C_{+}\right)$is detectable.

Combining Theorem 3.4 and Proposition 3.5, we obtain the following result. 
Corollary 3.6. If the Hautus test (3.9) is satisfied, then $(A, C)$ is detectable via the stabilizing output injection operator $L$ defined by (3.5).

Remark 3.7. Classically, the stabilizing output injection operator $L^{+}$of the finite dimensional projected system $\left(A^{+}, C^{+}\right)$can be determined by solving a (finite dimensional) Riccati equation. It is worth noticing that the matrices $A^{+}$and $C^{+}$are in practice of small size (their dimensions are respectively $\operatorname{dim} X_{+} \times \operatorname{dim} X_{+}$and $\operatorname{dim} Y_{+} \times \operatorname{dim} X_{+}$, with $\left.\operatorname{dim} Y_{+} \leqslant \operatorname{dim} X_{+}\right)$, making the computation of the Riccati matrix affordable.

Remark 3.8. A natural question is to determine the minimal number of effective outputs needed to stabilize an infinite dimensional system $A$ having a finite number of unstable eigenvalues. This issue has been investigated for the dual problem of stabilization of parabolic infinite dimensional systems by finite dimensional controls. For the boundary control of a linear parabolic equation, Triggiani has shown in [65] that this number is $\max _{k=1, \ldots, M} m_{k}^{\mathrm{G}}$ provided that the family $\left(C \varphi_{k, i}\right)_{1 \leqslant k \leqslant M, 1 \leqslant i \leqslant m_{k}^{\mathrm{G}}}$ is linearly independent (here $\left(\varphi_{k, i}\right)_{i \in\left\{1, \ldots, m_{k}^{\mathrm{G}}\right\}}$ denotes a basis of $\operatorname{Ker}\left(A-\lambda_{k}\right)$ and $m_{k}^{\mathrm{G}}$ is the geometric multiplicity of $\left.\lambda_{k}\right)$. More recently, Badra and Takahashi $[9,10]$ have proved for nonlinear parabolic infinite dimensional systems, that this number is indeed $\max _{k=1, \ldots, M} m_{k}^{\mathrm{G}}$ (even when the linear independence assumption fails). In particular, for an infinite dimensional system having simple eigenvalues, a one dimensional stabilizing feedback can be constructed.

\subsection{The diagonalizable case}

The rest of this section is devoted to the particular but important case where the unstable system is diagonalizable. Some of the results given here can be found in Barbu and Triggiani [12]. We assume that $A_{+}:=\left.A\right|_{\mathcal{D}(A) \cap X_{+}}$ is diagonalizable, i.e. $m_{k}^{\mathrm{G}}=m_{k}^{\mathrm{A}}$ for all unstable eigenvalues $\lambda_{k}$ of $A$. This implies in particular that the unstable space is $X_{+}=\bigoplus_{k=1}^{M} \operatorname{Ker}\left(A-\lambda_{k}\right)$, since $m_{k}^{\mathrm{P}}=1$, for all $k=1, \ldots, M$.

We denote by $N$ the number of unstable eigenvalues of $A$ counted with multiplicities. For the sake of simplicity, these unstable eigenvalues are still denoted $\lambda_{k}, k=1, \ldots, N$. We denote then by $\left(\varphi_{k}\right)_{1 \leqslant k \leqslant N}$ a basis of $X_{+}$. The eigenvalues of $A^{*}$ are given by the complex conjugates $\overline{\lambda_{k}}$ of the eigenvalues $\lambda_{k}$ of $A$ and have the same algebraic and geometric multiplicity. Denote by $\psi_{k}$ an eigenfunction of $A^{*}$ corresponding to the unstable eigenvalue $\overline{\lambda_{k}}$ $(1 \leqslant k \leqslant N)$. It can be shown (see [12], p. 1453) that the family $\left(\psi_{k}\right)_{1 \leqslant k \leqslant N}$ can be chosen such that $\left(\varphi_{k}\right)_{1 \leqslant k \leqslant N}$ and $\left(\psi_{k}\right)_{1 \leqslant k \leqslant N}$ form bi-orthogonal sequences, in the sense that $\left(\varphi_{k}, \psi_{m}\right)_{X}=\delta_{k m}$. It follows then that the projection operator $P_{+} \in \mathcal{L}\left(X, X_{+}\right)$defined in (3.1) can be expressed as

$$
P_{+} z=\sum_{k=1}^{N}\left(z, \psi_{k}\right)_{X} \varphi_{k} \quad(z \in X) .
$$

Since

$$
X_{+}=P_{+} X=\operatorname{Span}\left\{\varphi_{k}, 1 \leqslant k \leqslant N\right\}
$$

it follows that

$$
Y_{+}=C X_{+}=\operatorname{Span}\left\{C \varphi_{k}, 1 \leqslant k \leqslant N\right\}
$$

Assume now that the family

$$
\left(C \varphi_{k}\right)_{1 \leqslant k \leqslant N} \text { is linearly independent in } X \text {. }
$$

According to Claim 3.3. of [12], page 1458, this assumption holds true for an infinite dimensional system described by an evolution PDE with internal observation (this assertion will be proved in Lemma 4.4 in the setting of our population problem). Also note that it implies that the Hautus test (3.9) is necessarily satisfied and that:

$$
\operatorname{dim} Y_{+}=\operatorname{dim} X_{+}=N .
$$


We denote by $\mathbb{G}$ the Hermitian matrix of size $N \times N$ defined by

$$
\mathbb{G}=\left(\left(C \varphi_{i}, C \varphi_{j}\right)_{Y}\right)_{1 \leqslant i \leqslant N, 1 \leqslant j \leqslant N} .
$$

It is not difficult to prove that (3.10) is equivalent to the fact that $\mathbb{G}$ is invertible. We can then express the orthogonal projection operator $Q_{+}: Y \rightarrow Y_{+}:=C X_{+}$as follows (note that $\left(C \varphi_{k}\right)_{1 \leqslant k \leqslant N}$ is not an orthogonal family).

Lemma 3.9. Let $\mathbb{G}$ be the matrix defined by (3.11) and assume that property (3.10) holds true. Then, for any $y \in Y, Q_{+} y$ is defined by

$$
Q_{+} y=\sum_{i=1}^{N}\left(y, \eta_{i}\right)_{Y} C \varphi_{i}
$$

where

$$
\eta_{i}=\sum_{j=1}^{N} \alpha_{i j} C \varphi_{j}
$$

and

$$
\left(\alpha_{i j}\right)_{1 \leqslant i \leqslant N, 1 \leqslant j \leqslant N}=\mathbb{G}^{-1} .
$$

Proof. Recall that $Q_{+} y \in Y^{+}$is characterized by

$$
\forall y \in Y, \forall n \in\{1, \ldots, N\}, \quad\left(Q_{+} y-y, C \varphi_{n}\right)_{Y}=0 .
$$

Noting that $\left(\overline{\alpha_{i j}}\right)_{1 \leqslant i \leqslant N, 1 \leqslant j \leqslant N}$ is hermitian (because so is $\mathbb{G}$ ), we obtain

$$
\begin{aligned}
\left(\sum_{i=1}^{N}\left(y, \eta_{i}\right)_{Y} C \varphi_{i}, C \varphi_{n}\right) & =\sum_{j=1}^{N}\left(\sum_{i=1}^{N} \overline{\alpha_{i j}} \mathbb{G}_{i n}\right)\left(y, C \varphi_{j}\right) \\
& =\sum_{j=1}^{N}\left(\sum_{i=1}^{N} \alpha_{j i} \mathbb{G}_{i n}\right)\left(y, C \varphi_{j}\right) \\
& =\sum_{j=1}^{N} \delta_{j n}\left(y, C \varphi_{j}\right) \\
& =\left(y, C \varphi_{n}\right)
\end{aligned}
$$

and (3.12) is thus proved.

The (finite dimensional) operator $C_{+} \in \mathcal{L}\left(X_{+}, Y_{+}\right)$defined by (3.4) satisfies $C_{+} \varphi_{k}=C \varphi_{k}$ for any $k \in\{1, \ldots, N\}$. Note that $C_{+}$is nothing but the identity matrix when we choose as basis for $X_{+}$and $Y_{+}$ respectively $\left(\varphi_{k}\right)_{1 \leqslant k \leqslant N}$ and $\left(C \varphi_{k}\right)_{1 \leqslant k \leqslant N}$. Therefore, using these bases, $A_{+}+L_{+} C_{+}$is a Hurwitz matrix provided $\operatorname{diag}\left(\lambda_{1}, \ldots, \lambda_{N}\right)+L_{+}$is Hurwitz. It is thus sufficient to take $L_{+}=-\sigma I$ with

$$
\sigma>\operatorname{Re} \lambda_{1}
$$

to ensure the stability of $A_{+}+L_{+} C_{+}$.

The corresponding operator $L \in \mathcal{L}(Y, X)$ defined by (3.5) satisfies, for every $y \in Y$

$$
L y=L_{+} Q_{+} y=L_{+}\left(\sum_{i=1}^{N}\left(y, \eta_{i}\right)_{Y} C \varphi_{i}\right)=-\sigma \sum_{i=1}^{N}\left(y, \eta_{i}\right)_{Y} \varphi_{i},
$$

and Theorem 3.4 implies that $A+L C$ generates an exponentially stable semigroup. We summarize this useful result in the following Corollary. 
Corollary 3.10. Assume that $A_{+}:=\left.A\right|_{\mathcal{D}(A) \cap X_{+}}$is diagonalizable and let $N$ be the number of unstable eigenvalues of $A$ counted with multiplicities. We denote by $\left(\varphi_{i}\right)_{1 \leqslant i \leqslant N}$ a basis of $X_{+}$and we assume that the family $\left\{C \varphi_{i}, 1 \leqslant i \leqslant N\right\}$ is linearly independent in $X$. Given $\sigma>\operatorname{Re} \lambda_{1}$, let $L \in \mathcal{L}(Y, X)$ be defined by

$$
L y=-\sigma \sum_{i=1}^{N}\left(y, \eta_{i}\right)_{Y} \varphi_{i},
$$

where $\eta_{i}, i=1, \ldots, N$, is defined in Lemma 3.9. Then, $A+L C$ generates an exponentially stable semigroup on $X$.

\section{Application: Detectability And observer Design FOR THE POPUlation DYNAMICS SYSTEM}

Going back to the population system (1.1), our aim is to recover the distribution density of the population $p(a, x, t)$ for arbitrary $a$ and $x$ and for $t$ large enough, from the knowledge of $p(a, x, t)$ for arbitrary $t$ but only for an age interval $\left(a_{1}, a_{2}\right)$ (where $0 \leqslant a_{1}<a_{2} \leqslant a^{*}$ ) and a subdomain $\mathcal{O} \subset \Omega$. In other words, the available output is $y(t)=\left.p\right|_{\left(a_{1}, a_{2}\right) \times \mathcal{O}}$ where $t \in(0, T)$ ( $T$ has to be chosen large enough). At the end of this section, we will see that more realistic outputs can also be considered, such as space locally distributed total population and pointwise observation in age (see Rems. 4.5 and 4.6).

To achieve this, we will construct an observer for system (1.1). Observers have been introduced by Luenberger to estimate the state of a finite dimensional dynamical system from the knowledge of an output (of course assuming that the initial state is unknown). Roughly speaking, an observer is an auxiliary dynamical system that uses as inputs the available measurements (that is the output of the original system) and converges asymptotically (in time) towards the state of the original system. The generalization of observers (also called estimators or filters in the stochastic framework) to infinite dimensional systems goes to the eighties (see for instance Baras et al. [11,35], Chapman and Pritchard [15], Curtain and Zwart [18] and Hidayat et al. [32] for a brief overview of observers in the context of distributed systems). Observers have been successfully used to solve data assimilation problems in geophysics (see [4-6]), initial data reconstruction (see $[24,30,31,54]$ ) and joint state and/or parameter identification by Curtain, Demetriou and Ito [19], Moireau et al. [14,46,47], Krstic et al. $[40,58]$.

Using the notation of Section 2, let $A$ be defined by (2.3) and (2.4). We consider the abstract system (2.7), i.e.

with the output

$$
\dot{p}(t)=A p(t), \quad t \in(0, T),
$$

$$
y(t)=C p(t), \quad t \in(0, T),
$$

where the observation operator $C \in \mathcal{L}(X, Y)$, with $Y:=L^{2}\left(\left(a_{1}, a_{2}\right) \times \mathcal{O}\right)$ is defined by

$$
C \varphi=\left.\varphi\right|_{\left(a_{1}, a_{2}\right) \times \mathcal{O}} \quad(\varphi \in X) .
$$

We introduce the observer

$$
\left\{\begin{array}{l}
\dot{\hat{p}}(t)=A \widehat{p}(t)+L C \widehat{p}(t)-L y(t), \quad t \in(0, T), \\
\widehat{p}(0)=0
\end{array}\right.
$$

where $L \in \mathcal{L}(Y, X)$ is a linear operator to be defined. As explained in Section 1, the error $e:=\widehat{p}-p$ satisfies

$$
\left\{\begin{array}{l}
\dot{e}(t)=(A+L C) e(t), \quad t \in(0, T), \\
e(0)=-p(0) .
\end{array}\right.
$$

According to Remark 2.5 and Proposition 2.6, assumptions (A.1) and (A.2) (see the beginning of Sect. 3) are satisfied for the above system. Therefore, the problem of determining the stabilizing output injection operator $L$ for $(A, C)$ fits into the framework described in Section 3 and we can apply the approach developed 
therein. Denoting by $\Sigma_{+}$the set of the eigenvalues of $A$ with non negative real part, it only remains to verify the assumption of Proposition 3.5, and in particular the following lemma shows that the Hautus test of Proposition 3.5 is satisfied for our system $(A, C)$.

Lemma 4.1. If $\varphi \in \mathcal{D}(A)$ satisfies $A \varphi=\lambda \varphi$ for $\lambda \in \Sigma_{+}$and $C \varphi=0$, then $\varphi$ vanishes identically.

Proof. Let $\lambda$ be an unstable eigenvalue of $A$ (i.e. $\operatorname{Re} \lambda \geqslant 0)$ and let $\varphi \in \mathcal{D}(A)$ satisfying $A \varphi=\lambda \varphi$. Decomposing $\varphi(0, x)$ in the basis of $L^{2}(\Omega)$ constituted of the eigenfunctions of the Dirichlet Laplacian (more precisely, of $-k \Delta)$, one can easily check that the unique solution of the evolution system

$$
\begin{cases}\frac{\partial \varphi}{\partial a}(a, x)=k \Delta \varphi(a, x)-(\lambda+\mu) \varphi(a, x), & a \in\left(0, a^{*}\right), x \in \Omega, \\ \varphi(a, x)=0, & a \in\left(0, a^{*}\right), x \in \partial \Omega, \\ \varphi(0, x)=\sum_{j \in \mathbb{N}} \alpha_{j} \varphi_{j}^{D}(x), & x \in \Omega,\end{cases}
$$

is given by

$$
\varphi(a, x)=\sum_{j \in \mathbb{N}} \alpha_{j} \mathrm{e}^{-\left(\lambda+\lambda_{j}^{D}\right) a} \Pi(a) \varphi_{j}^{D}(x) .
$$

Plugging the above expression in the renewal equation (the last equation of (1.1)), we obtain

$$
\sum_{j \in \mathbb{N}} \alpha_{j} \varphi_{j}^{D}(x)=\sum_{j \in \mathbb{N}} \alpha_{j}\left(\int_{0}^{a^{*}} \beta(a) \mathrm{e}^{-\left(\lambda+\lambda_{j}^{D}\right) a} \Pi(a)\right) \varphi_{j}^{D}(x) .
$$

We see that is equivalent to, for any $j \in \mathbb{N}$, either $\alpha_{j}=0$, either $\lambda+\lambda_{j}^{D}$ solves the characteristic equation (2.2) of the diffusion free problem. Consequently, we have

$$
\varphi(a, x)=\sum_{j \mid \lambda+\lambda_{j}^{D} \in \sigma\left(A_{0}\right)} \alpha_{j} \mathrm{e}^{-\left(\lambda+\lambda_{j}^{D}\right) a} \Pi(a) \varphi_{j}^{D}(x) .
$$

The condition $C \varphi=0$ reads then

$$
\left.\sum_{j \mid \lambda+\lambda_{j}^{D} \in \sigma\left(A_{0}\right)} \alpha_{j} \mathrm{e}^{-\left(\lambda+\lambda_{j}^{D}\right) a} \varphi_{j}^{D}\right|_{\mathcal{O}}(x)=0, \quad a \in\left(a_{1}, a_{2}\right) .
$$

Since the eigenfunctions of $-k \Delta$ with Dirichlet boundary conditions are analytic, we immediately obtain that $\varphi=0$.

Remark 4.2. The above proof shows in particular that the result still holds when the diffusion coefficient depends analytically on age.

Applying Corollary 3.6 to (4.3) immediately leads to the following result.

Theorem 4.3. Let $p_{0} \in X$ and $p$ the solution of (1.1). Assume that $y(t)=\left.p\right|_{\left(a_{1}, a_{2}\right) \times \mathcal{O}}(t>0)$ is known. Let $\widehat{p}$ the observer defined by (4.2), where $L \in \mathcal{L}(Y, X)$ is the (finite dimensional) stabilizing output injection operator defined by (3.5). Then, there exist $C>0$ and $\omega>0$ such that

$$
\|\widehat{p}(t)-p(t)\| \leqslant C \mathrm{e}^{-\omega t}\left\|p_{0}\right\|, \quad t>0 .
$$


To conclude this Section, we go back to the particular but important case where the unstable system is diagonalizable (see Sect. 3.2), i.e. we assume that $A_{+}:=\left.A\right|_{\mathcal{D}(A) \cap X_{+}}$is diagonalizable. For the sake of simplicity, we denote here by $M$ the number of unstable eigenvalues of $A$ counted without multiplicities (still denoted $\lambda_{k}$, $k=1, \ldots, M)$ and we denote by $\left(\varphi_{k, i}\right)_{i \in\left\{1, \ldots, m_{k}^{\mathrm{G}}\right\}}$ a basis of $\operatorname{Ker}\left(A-\lambda_{k}\right)$ where $m_{k}^{\mathrm{G}}$ is the geometric multiplicity of $\lambda_{k}(k=1, \ldots, M)$. In order to show that the stabilizing output injection operator $L$ can be rewritten as (3.16), it remains to verify that assumption (3.10) holds. This is done in the following lemma, which is exactly Part (A) of Claim 3.3. in ([12], p. 1458). The proof is recalled here for reader's convenience.

Lemma 4.4. The family $\left(C \varphi_{k, i}\right)_{1 \leqslant k \leqslant M, 1 \leqslant i \leqslant m_{k}^{\mathrm{G}}}$ is linearly independent in $X$.

Proof.

Step 1. It is easy to prove that the Hautus test (3.9), which is verified in Lemma 4.1 for $A$ and $C$ respectively defined by $(2.3)-(2.4)$ and $(4.1)$, is equivalent to the fact that

$$
\forall k \in\{1, \ldots, M\}, \text { the family }\left(C \varphi_{k, i}\right)_{1 \leqslant i \leqslant m_{k}^{G}} \text { is linearly independent in } X .
$$

Step 2. Consider now the case of two distinct eigenvalues, say $\lambda_{1}$ and $\lambda_{2}$. Assume by contradiction that the corresponding system of eigenfunctions

$$
\left\{C \varphi_{1, i}, C \varphi_{2, j} \mid 1 \leqslant i \leqslant m_{1}^{\mathrm{G}}, 1 \leqslant j \leqslant m_{2}^{\mathrm{G}}\right\}
$$

is linearly dependent and assume, for instance, that

$$
C \varphi_{2, m_{2}^{\mathrm{G}}}=\sum_{i=1}^{m_{1}^{\mathrm{G}}} \alpha_{i} C \varphi_{1, i}+\sum_{j=1}^{m_{2}^{\mathrm{G}}-1} \beta_{j} C \varphi_{2, j}
$$

i.e. that

$$
\varphi_{2, m_{2}^{\mathrm{G}}}=\sum_{i=1}^{m_{1}^{\mathrm{G}}} \alpha_{i} \varphi_{1, i}+\sum_{j=1}^{m_{2}^{\mathrm{G}}-1} \beta_{j} \varphi_{2, j} \quad \text { on }\left(a_{1}, a_{2}\right) \times \mathcal{O} .
$$

Applying the differential operator $A=-\partial_{a}-\mu \cdot+k \Delta$ defined by $(2.4)$, we obtain that on $\left(a_{1}, a_{2}\right) \times \mathcal{O}$ :

$$
0=A\left(\varphi_{2, m_{2}^{\mathrm{G}}}-\sum_{i=1}^{m_{1}^{\mathrm{G}}} \alpha_{i} \varphi_{1, i}-\sum_{j=1}^{m_{2}^{\mathrm{G}}-1} \beta_{j} \varphi_{2, j}\right)=\lambda_{2} \varphi_{2, m_{2}^{\mathrm{G}}}-\lambda_{1} \sum_{i=1}^{m_{1}^{\mathrm{G}}} \alpha_{i} \varphi_{1, i}-\lambda_{2} \sum_{j=1}^{m_{2}^{\mathrm{G}}-1} \beta_{j} \varphi_{2, j}
$$

which implies, using again (4.4) that

$$
\left(\lambda_{2}-\lambda_{1}\right) \sum_{i=1}^{m_{1}^{\mathrm{G}}} \alpha_{i} \varphi_{1, i}=0 \quad \text { on }\left(a_{1}, a_{2}\right) \times \mathcal{O} .
$$

Using the fact that $\lambda_{1} \neq \lambda_{2}$ and Step 1 , we find that $\alpha_{i}=0$ for all $i \in\left\{1, \ldots, m_{1}^{G}\right\}$. Therefore $\varphi_{2, m_{2}^{G}}=$ $\sum_{j=1}^{m_{2}^{\mathrm{G}}-1} \beta_{j} \varphi_{2, j}$ on $\left(a_{1}, a_{2}\right) \times \mathcal{O}$, which contradicts Step 1 .

Step 3. Finally we prove the statement of Lemma 4.4 by induction with respect to the number $q \leqslant M$ of distinct eigenvalues $\lambda_{k}$. Assume that the statement holds for $q-1$ distinct eigenvalues $\lambda_{1}, \ldots, \lambda_{q-1}$ and consider the system $\left(C \varphi_{k, i}\right)_{1 \leqslant k \leqslant q, 1 \leqslant i \leqslant m_{k}^{G}}$. By contradiction, assume that this system is linearly dependent. As in Step 2, we may assume, for instance, that

$$
\varphi_{q, m_{q}^{\mathrm{G}}}=\sum_{k=1}^{q-1} \sum_{i=1}^{m_{k}^{\mathrm{G}}} \alpha_{k i} \varphi_{k, i}+\sum_{j=1}^{m_{q}^{\mathrm{G}}-1} \beta_{j} \varphi_{q, j} \quad \text { on }\left(a_{1}, a_{2}\right) \times \mathcal{O} .
$$


Applying the differential operator $A$ and proceeding as in Step 2, we obtain that

$$
\sum_{k=1}^{q-1}\left(\lambda_{q}-\lambda_{k}\right) \sum_{i=1}^{m_{k}^{\mathrm{G}}} \alpha_{k i} \varphi_{k, i}=0 \quad \text { on }\left(a_{1}, a_{2}\right) \times \mathcal{O} .
$$

As the eigenvalues are distinct, by the inductive hypothesis, this implies that $\alpha_{k i}=0$ for any $k \in\{1, \ldots, q-1\}$ and $i \in\left\{1, \ldots, m_{k}^{\mathrm{G}}\right\}$. Therefore

$$
\varphi_{q, m_{q}^{\mathrm{G}}}=\sum_{j=1}^{m_{q}^{\mathrm{G}}-1} \beta_{j} \varphi_{q, j} \quad \text { on }\left(a_{1}, a_{2}\right) \times \mathcal{O},
$$

which contradicts Step 1 and ends the proof.

The results in this section can be extended to other observation operators, as shown in the two following remarks.

Remark 4.5. Given $a_{1}, a_{2}$ with $0 \leqslant a_{1}<a_{2} \leqslant a^{*}$, we can take $Y=L^{2}(\mathcal{O})$ and $C \in \mathcal{L}(X, Y)$ defined by

$$
(C \varphi)(x)=\int_{a_{1}}^{a_{2}} \varphi(a, x) \mathrm{d} a, \quad \varphi \in X, x \in \mathcal{O} .
$$

Indeed, using the third assertion in Theorem 2.4, it follows that if $\varphi$ satisfies $A \varphi=\lambda \varphi$ for some $\lambda \in \mathbb{C}$ and $C \varphi=0$ then there exist complex numbers $\alpha_{j}$ such that

$$
\sum_{j \mid \lambda+\lambda_{j}^{D} \in \sigma\left(A_{0}\right)} \alpha_{j} \varphi_{j}^{D}(x) \int_{a_{1}}^{a_{2}} \mathrm{e}^{-\lambda_{i(j)}^{0} a} \Pi(a) \mathrm{d} a=0, \quad x \in \mathcal{O},
$$

where $i(j) \in \mathbb{N}^{*}$ is the unique index such that $\lambda_{i}^{0}=\lambda+\lambda_{j}^{D}$. Using again the analyticity of the functions $\varphi_{j}^{D}$, combined with the fact that $\mathrm{e}^{-\lambda_{i(j)}^{0} a} \Pi(a)>0$ for $a \in\left[a_{1}, a_{2}\right]$, we conclude that $\varphi=0$. The observation operator defined in (4.5) is clearly of biological interest since measuring $y=C p$ signifies counting the population having an age in the interval $\left(a_{1}, a_{2}\right)$ and localized in the spatial domain $\mathcal{O}$.

Remark 4.6. Another possible choice of the observation operator, which can be seen as a limit case of the operator defined in (4.1) is

$$
(C \varphi)(x)=\varphi\left(a_{0}, x\right), \quad \varphi \in X, x \in \mathcal{O},
$$

where $a_{0} \in\left(0, a^{*}\right)$ is a fixed age. In this case, the conditions $A \varphi=\lambda \varphi$ for some $\lambda \in \mathbb{C}$ and $C \varphi=0$ write

$$
\begin{gathered}
-\partial_{a} \varphi-\mu \varphi+k \Delta \varphi=\lambda \varphi, \quad \varphi \in \mathcal{D}(A), \\
\varphi\left(a_{0}, x\right)=0, \quad x \in \mathcal{O} .
\end{gathered}
$$

Looking to the first equation in (4.7) as a parabolic equation with $a$ in the role of the time variable and applying a well-known unique continuation result for parabolic equations (see, for instance, [50], Prop. 2.2) we obtain that $\varphi=0$. Note that the argument used to show the observability of eigenfunctions in this case (and, consequently, for the observation operator (4.1)) can be used, without any modification, in the case $\mu=\mu(a, x)$ and $\beta=\beta(a, x)$, as assumptions (A.1) and (A.2) are satisfied in this case (see for instance [26]). 


\section{Discretization}

From now on, we assume for the sake of simplicity that $\Omega$ is the interval $(0, \ell)$, that $A_{+}:=\left.A\right|_{\mathcal{D}(A) \cap X_{+}}$is diagonalizable and that $A$ has $N$ unstable eigenvalues $\lambda_{1}, \ldots, \lambda_{N}$ counted with multiplicities.

Our goal in the last two sections is to show how to implement the proposed method to estimate the final state from partial observations. More precisely, assuming that $p_{0}$ is an unknown initial data, we want to estimate $p$ at time $t=T$ where $p$ solves the following open loop system:

$$
\begin{cases}\partial_{t} p(a, x, t)+\partial_{a} p(a, x, t)+\mu(a) p(a, x, t)-k \partial_{x x} p(a, x, t)=0, & a \in\left(0, a^{*}\right), x \in(0, \ell), t>0, \\ p(a, 0, t)=p(a, \ell, t)=0, & a \in\left(0, a^{*}\right), t>0, \\ p(a, x, 0)=p_{0}(a, x), & a \in\left(0, a^{*}\right), x \in(0, \ell), \\ p(0, x, t)=\int_{0}^{a^{*}} \beta(a) p(a, x, t) \mathrm{d} a, & x \in(0, \ell), t>0,\end{cases}
$$

provided we know the observation

$$
y(t)=p(t)_{\mid\left(a_{1}, a_{2}\right) \times\left(\ell_{1}, \ell_{2}\right)}, \quad t \in(0, T),
$$

where $0 \leqslant a_{1}<a_{2} \leqslant a^{*}$ and $0 \leqslant \ell_{1}<\ell_{2} \leqslant \ell$.

This leads to define the observation operator $C \in \mathcal{L}(X, Y)$

$$
C \varphi=\varphi_{\mid\left(a_{1}, a_{2}\right) \times\left(\ell_{1}, \ell_{2}\right)}, \quad \forall \varphi \in X
$$

where $X=L^{2}\left(\left(0, a^{*}\right) \times(0, \ell)\right)$ and $Y=L^{2}\left(\left(a_{1}, a_{2}\right) \times\left(\ell_{1}, \ell_{2}\right)\right)$.

In order to estimate $p(T)$, we use the observer designed in Section 4. As the unstable space is the $N$-dimensional space (see Sect. 2),

$$
\begin{aligned}
X_{+}=\bigoplus_{k=1}^{N} \operatorname{Ker}\left(A-\lambda_{k}\right) & =\operatorname{Span}\left\{\varphi_{k}, 1 \leqslant k \leqslant N\right\} \\
& =\operatorname{Span}\left\{\varphi_{i}^{0}(a) \varphi_{j}^{D}(x), \lambda_{i}^{0}-\lambda_{j}^{D}=\lambda_{k}, 1 \leqslant k \leqslant N\right\}
\end{aligned}
$$

the observer defined by (4.2) involves the stabilizing output injection operator $L$ defined by (3.16) (see Sect. 3.2)

$$
L y=-\sigma \sum_{i=1}^{N}\left(y, \eta_{i}\right)_{Y} \varphi_{i} \quad(y \in Y),
$$

where $\sigma>\lambda_{1}$ (gain coefficient) and

$$
\eta_{i}=\sum_{j=1}^{N} \alpha_{i j} C \varphi_{j} \quad \text { and } \quad\left(\alpha_{i j}\right)_{1 \leqslant i \leqslant N, 1 \leqslant j \leqslant N}=\mathbb{G}^{-1} .
$$

(see (3.13) and (3.14)).

The observer solves then the following system

$$
\begin{cases}\partial_{t} \widehat{p}(a, x, t)+\partial_{a} \widehat{p}(a, x, t)+\mu(a) \widehat{p}(a, x, t)-k \partial_{x x} \widehat{p}(a, x, t) & \\ \quad+\sigma \sum_{i=1}^{N}\left(C \widehat{p}, \eta_{i}\right)_{Y} \varphi_{i}(a, x)=\sigma \sum_{i=1}^{N}\left(y, \eta_{i}\right)_{Y} \varphi_{i}(a, x), & a \in\left(0, a^{*}\right), x \in(0, \ell), t>0, \\ \widehat{p}(a, 0, t)=\widehat{p}(a, \ell, t)=0, & a \in\left(0, a^{*}\right), t>0, \\ \widehat{p}(a, x, 0)=0, & a \in\left(0, a^{*}\right), x \in(0, \ell), \\ \widehat{p}(0, x, t)=\int_{0}^{a^{*}} \beta(a) \widehat{p}(a, x, t) \mathrm{d} a, & x \in(0, \ell), t>0 .\end{cases}
$$


Numerical approximation of population models with spatial diffusion (typically of the form (5.1)) has been considered in several papers. Lopez and Trigiante proposed in [43] an adaptative finite difference scheme to handle the singularity of the mortality function $\mu$ as $a \rightarrow a^{*}$. Milner [45] and Kim [38] proposed schemes using finite difference along the characteristic age-time direction and finite elements in space or mixed finite element [39]. Huyer [33] developed a semigroup approach to discretize the problem using a Galerkin approximation involving Laguerre polynomials in age and spectral decomposition in space. Ayati [7] proposed a method allowing variable time steps and independent age and time discretizations. Ayati and Dupont [8] proposed Galerkin approximations in both age and space for a population model with nonlinear diffusion. Let us also mention the contributions of Gerardo-Giorda et al. who proposed approximations using finite difference in age and time and finite element in space for an age structured model with space and age dependent diffusion in [20] and density dependent diffusion in [25]. We refer the interested reader to the Ph.D. of Pelovska [48] for more references.

Below, we successively present finite difference discretizations of systems (5.1) and (5.2). For both problems, one of the main difficulties comes from the discretization of the renewal equation $p(0, x, t)=\int_{0}^{a^{*}} \beta(a) p(a, x, t) \mathrm{d} a$ appearing in the domain of the operator. Indeed, this non local equation couples the unknowns corresponding to different ages for a given time and at a given point of space. In [33], Huyer overcame this problem by proposing a reformulation of the system in which this condition is taken into account by adding a Dirac term in the evolution PDE defining the dynamics. Here, we use the same approach as in [20], in which the renewal equation is simplified via the time discretization: the density of population at age 0 for a given time $t^{n}$ is evaluated using the values of the density for all ages at the previous time $t^{n-1}$ (see Fig. 3).

\subsection{Discretization of the open loop problem: data generation}

\subsubsection{Rescaling the problem}

In order to overcome the difficulties due to singular behavior of the coefficient $\mu$ (see Eq. (1.3)), we follow [20] and introduce the auxiliary variable

$$
u(a, x, t)=\frac{p(a, x, t)}{\Pi(a)}=\exp \left(\int_{0}^{a^{*}} \mu(s) \mathrm{d} s\right) p(a, x, t) .
$$

One can easily check that $u$ satisfies

$$
\begin{cases}\partial_{t} u(a, x, t)+\partial_{a} u(a, x, t)-k \partial_{x x} u(a, x, t)=0, & a \in\left(0, a^{*}\right), x \in(0, \ell), t>0, \\ u(a, 0, t)=u(a, \ell, t)=0, & a \in\left(0, a^{*}\right), t>0, \\ u(a, x, 0)=u_{0}(a, x), & a \in\left(0, a^{*}\right), x \in(0, \ell), \\ u(0, x, t)=\int_{0}^{a^{*}} m(a) u(a, x, t) \mathrm{d} a, & x \in(0, \ell), t>0,\end{cases}
$$

where we have set $u_{0}(a, x)=p_{0}(a, x) / \Pi(a)$ and where $m(a)=\beta(a) \Pi(a)$ stands for the maternity function.

\subsubsection{Finite difference discretization in time}

Let $u^{n}(a, x)$ be an approximation of $u\left(a, x, t^{n}\right)$, where $t^{n}=n \Delta t, 0 \leqslant n \leqslant N_{t}, \Delta t=T / N_{t}$ is a discretization of $(0, T)$. Starting from $u^{0}(a, x)=u_{0}(a, x)$, we construct $u^{n}$ for $n \geqslant 1$ using an Euler's backwards scheme

$$
\begin{cases}\frac{1}{\Delta t}\left(u^{n}(a, x)-u^{n-1}(a, x)\right)+\partial_{a} u^{n}(a, x)-k \partial_{x x} u^{n}(a, x)=0, & a \in\left(0, a^{*}\right), x \in(0, \ell), \\ u^{n}(a, 0)=u^{n}(a, \ell)=0, & a \in\left(0, a^{*}\right), \\ u^{0}(a, x)=u_{0}(a, x), & a \in\left(0, a^{*}\right), x \in(0, \ell), \\ u^{n}(0, x)=\int_{0}^{a^{*}} m(a) u^{n-1}(a, x) \mathrm{d} a, & x \in(0, \ell) .\end{cases}
$$




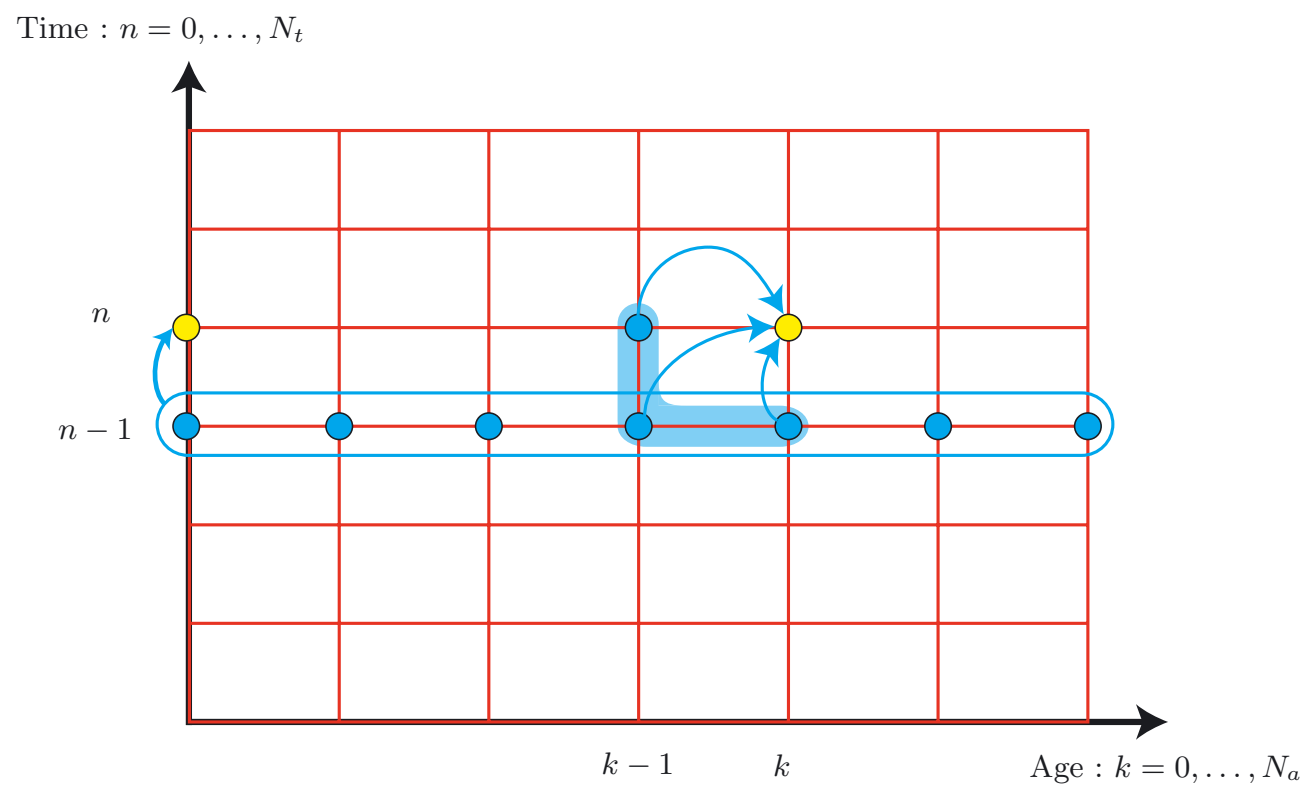

Figure 3. Step $n$ of the algorithm. The unknown $\mathbf{U}^{n, k}$ at each node $(k, n)$ is the vector containing the solution at the different points of the space discretization.

\subsubsection{Finite difference discretization in space}

Denoting by $u_{i}^{n}(a)$ an approximation of $u^{n}\left(x_{i}, a\right)$ (where $x_{i}=i h=i \ell /\left(N_{x}+1\right)$, with $\left.0 \leqslant i \leqslant N_{x}+1\right)$ and using a classical centered approximation for the second order derivative in space, the above system yields

$$
\left\{\begin{array}{l}
\frac{\mathrm{d} \mathbf{U}^{n}}{\mathrm{~d} a}(a)+\frac{1}{h^{2}} \mathbb{K} \mathbf{U}^{n}(a)+\frac{1}{\Delta t} \mathbf{U}^{n}(a)=\frac{1}{\Delta t} \mathbf{U}^{n-1}(a), \\
\mathbf{U}^{n}(0)=\int_{0}^{a^{*}} m(a) \mathbf{U}^{n-1}(a) \mathrm{d} a, \\
\mathbf{U}^{0}(a)=\mathbf{U}_{0}(a),
\end{array}\right.
$$

where $\mathbf{U}^{n}(a):=\left(u_{1}^{n}(a), \ldots, u_{N_{x}}^{n}(a)\right)^{\mathbf{T}}, \mathbf{U}_{0}(a):=\left(u_{0}\left(a, x_{1}\right), \ldots, u_{0}\left(a, x_{N_{x}}\right)\right)^{\mathbf{T}}$ and

$$
\mathbb{K}=k\left(\begin{array}{ccccccc}
2 & -1 & & & & \\
-1 & 2 & -1 & & 0 & \\
& -1 & 2 & -1 & & \\
& & \ddots & \ddots & \ddots & \\
& 0 & & -1 & 2 & -1 \\
& & & & -1 & 2
\end{array}\right) .
$$

\subsubsection{Finite difference discretization in age}

We use a Crank-Nicholson scheme to approximate the solution of problem (5.3). Denoting by $u_{i}^{n, k}$ an approximation of $u_{i}^{n}\left(a^{k}\right)$, where $a^{k}=k \Delta a, 0 \leqslant k \leqslant N_{a}, \Delta a=a^{*} / N_{a}$, and by $\mathbf{U}^{n, k}=\left(u_{1}^{n, k}, \ldots, u_{N_{x}}^{n, k}\right)^{\mathbf{T}}$ an approximation of $\mathbf{U}^{n}\left(a^{k}\right)$, we move from age $a^{k-1}$ to age $a^{k}$ following the scheme

$$
\frac{1}{\Delta a}\left(\mathbf{U}^{n, k}-\mathbf{U}^{n, k-1}\right)+\frac{1}{2 h^{2}} \mathbb{K}\left(\mathbf{U}^{n, k}+\mathbf{U}^{n, k-1}\right)+\frac{1}{2 \Delta t}\left(\mathbf{U}^{n, k}+\mathbf{U}^{n, k-1}\right)=\frac{1}{2 \Delta t}\left(\mathbf{U}^{n-1, k}+\mathbf{U}^{n-1, k-1}\right),
$$


with the initial conditions

$$
\left\{\begin{array}{l}
\mathbf{U}^{0, k}=\mathbf{U}_{0}\left(a^{k}\right), \quad \forall k=0, \ldots, N_{a} \\
\mathbf{U}^{n, 0}=\sum_{k=0}^{N_{a}} \omega_{k} m\left(a^{k}\right) \mathbf{U}^{n-1, k} \simeq \int_{0}^{a^{*}} m(a) \mathbf{U}^{n-1}(a) \mathrm{d} a,
\end{array}\right.
$$

where $\omega_{k}$ are the weights of the quadrature formula used.

\subsubsection{Algorithm for the open loop system}

(1) Initialization for $n=0: \mathbf{U}^{0, k}=\left(u_{0}\left(k \Delta a, x_{1}\right), \ldots, u_{0}\left(k \Delta a, x_{N_{h}}\right)^{\mathbf{T}}\right.$

(2) For $n=1, \ldots, N_{t}$ :

- Initialization of $\mathbf{U}^{n, 0}$ using the values of $\left(\mathbf{U}^{n-1, j}\right): \mathbf{U}^{n, 0}=\sum_{k=0}^{N_{a}} \omega_{k} m\left(a^{k}\right) \mathbf{U}^{n-1, k}$.

- For $k=1, \ldots, N_{a}, \mathbf{U}^{n, k}=\left(u_{1}^{n, k}, \ldots, u_{N_{x}}^{n, k}\right)^{\mathbf{T}}$ solves the linear system

$$
\mathbb{A} \mathbf{U}^{n, k}=\mathbf{b}^{n, k}
$$

where

$$
\begin{gathered}
\mathbb{A}=\left(\Delta t+\frac{1}{2} \Delta a\right) \mathbb{I}+\frac{1}{2} \frac{\Delta t \Delta a}{h^{2}} \mathbb{K} \\
\mathbf{b}^{n, k}=\frac{1}{2} \Delta a\left(\mathbf{U}^{n-1, k}+\mathbf{U}^{n-1, k-1}\right)+\left[\left(\Delta t-\frac{1}{2} \Delta a\right) \mathbb{I}-\frac{1}{2} \frac{\Delta t \Delta a}{h^{2}} \mathbb{K}\right] \mathbf{U}^{n, k-1}
\end{gathered}
$$

- $\mathbf{P}^{n, k}=\left(p_{1}^{n, k}, \ldots, p_{N_{x}}^{n, k}\right)^{\mathbf{T}}$ where $p_{i}^{n, k}=\Pi\left(a^{k}\right) u_{i}^{n, k}$.

- $\mathbf{Y}^{n, k}=\left(y_{1}^{n, k}, \ldots, y_{N_{x}}^{n, k}\right)^{\mathbf{T}}$ where $y_{i}^{n, k}=p_{i}^{n, k}$ if $\ell_{1} \leqslant i h \leqslant \ell_{2}$ and $y_{i}^{n, k}=0$ otherwise.

\subsection{Discretization of the closed loop system: observer design}

In order to discretize (5.2), we follow the same procedure as in the previous subsection for the open loop system. The main differences in the dynamics are the presence of a source term coming from the observation $y$ and the extra term in the evolution equation $\sum_{i=1}^{N}\left(C \widehat{p}, \eta_{i}\right)_{Y} \varphi_{i}$.

\subsubsection{Rescaling the problem}

First of all, in order to overcome the difficulties due to singular behavior of the coefficient $\mu$, we introduce as we did for the open loop system, the auxiliary variable

$$
\widehat{u}(a, x, t)=\frac{\widehat{p}(a, x, t)}{\Pi(a)}=\exp \left(\int_{0}^{a^{*}} \mu(s) \mathrm{d} s\right) \widehat{p}(a, x, t),
$$

where $\widehat{p}$ solves (5.2). 
One can easily check that $\widehat{u}$ satisfies

$$
\begin{cases}\partial_{t} \widehat{u}(a, x, t)+\partial_{a} \widehat{u}(a, x, t)-k \partial_{x x} \widehat{u}(a, x, t) & \\ \quad+\sigma \sum_{i=1}^{N}\left(C \Pi \widehat{u}, \eta_{i}\right)_{Y} v_{i}(a, x)=\sigma \sum_{i=1}^{N}\left(y(t), \eta_{i}\right)_{Y} v_{i}(a, x), & a \in\left(0, a^{*}\right), x \in(0, \ell), t>0, \\ \widehat{u}(a, 0, t)=\widehat{u}(a, \ell, t)=0, & a \in\left(0, a^{*}\right), t>0, \\ \widehat{u}(a, x, 0)=0, & a \in\left(0, a^{*}\right), x \in(0, \ell), \\ \widehat{u}(0, x, t)=\int_{0}^{a^{*}} m(a) \widehat{u}(a, x, t) \mathrm{d} a, & x \in(0, \ell), t>0,\end{cases}
$$

where we have set $v_{i}(a, x)=\varphi_{i}(a, x) / \Pi(a)(1 \leqslant i \leqslant N)$.

In order to discretize the term $\left(C \Pi \widehat{u}, \eta_{i}\right)_{Y}$ in (5.4), let us introduce the new (scalar) unknowns

$$
\theta_{i}(t)=\left(C \Pi \widehat{u}, \eta_{i}\right)_{Y} \quad(1 \leqslant i \leqslant N) .
$$

Using the first equation in (5.4) and the fact that, for any $i, j \in\{1, \ldots, N\}$,

$$
\left(C \Pi v_{j}, \eta_{i}\right)_{Y}=\left(C \varphi_{j}, \eta_{i}\right)_{Y}=\sum_{k=1}^{N} \overline{\alpha_{i k}}\left(C \varphi_{j}, C \varphi_{k}\right)_{Y}=\sum_{k=1}^{N} \overline{\alpha_{i k}\left(C \varphi_{k}, C \varphi_{j}\right)_{Y}}=\delta_{i j}
$$

(by (3.13) and (3.14)), we remark that $\theta_{i}$ satisfies

$$
\dot{\theta}_{i}(t)=-\left(C \Pi \partial_{a} \widehat{u}, \eta_{i}\right)_{Y}+k\left(C \Pi \partial_{x x} \widehat{u}, \eta_{i}\right)_{Y}-\sigma \theta_{i}(t)+\sigma\left(y, \eta_{i}\right)_{Y} .
$$

Consequently, problem (5.4) can be reformulated as follows

$$
\begin{cases}\dot{\theta}_{i}(t)=-\left(C \Pi \partial_{a} \widehat{u}(t), \eta_{i}\right)_{Y}+k\left(C \Pi \partial_{x x} \widehat{u}(t), \eta_{i}\right)_{Y} & \\ \quad-\sigma \theta_{i}(t)+\sigma\left(y(t), \eta_{i}\right)_{Y}, & t>0,1 \leqslant i \leqslant N, \\ \partial_{t} \widehat{u}(a, x, t)+\partial_{a} \widehat{u}(a, x, t)-k \partial_{x x} \widehat{u}(a, x, t)+\sigma \sum_{i=1}^{N} \theta_{i}(t) v_{i}(a, x) & \\ \quad=\sigma \sum_{i=1}^{N}\left(y(t), \eta_{i}\right)_{Y} v_{i}(a, x), & a \in\left(0, a^{*}\right), x \in(0, \ell), t>0, \\ \theta_{i}(0)=0, & 1 \leqslant i \leqslant N, \\ \widehat{u}(a, 0, t)=\widehat{u}(a, \ell, t)=0, & a \in\left(0, a^{*}\right), t>0, \\ \widehat{u}(a, x, 0)=0, & a \in\left(0, a^{*}\right), x \in(0, \ell), \\ \widehat{u}(0, x, t)=\int_{0}^{a^{*}} m(a) \widehat{u}(a, x, t) \mathrm{d} a, & x \in(0, \ell), t>0 .\end{cases}
$$

\subsubsection{Finite difference discretization in time}

Let $\widehat{u}^{n}(a, x)\left(\right.$ resp. $\left.\theta_{i}^{n}, y^{n}(a, x)\right)$ be an approximation of $\widehat{u}\left(a, x, t^{n}\right)\left(\right.$ resp. $\left.\theta_{i}\left(t^{n}\right), y\left(a, x, t^{n}\right)\right)$, where $t^{n}=n \Delta t$, $0 \leqslant n \leqslant N_{t}, \Delta t=T / N_{t}$ is a discretization of $(0, T)$. Starting from $\theta_{i}^{0}=0$ and $\widehat{u}^{0}(a, x)=0$, we construct $\theta_{i}^{n}$ 
and $\widehat{u}^{n}$ for $n \geqslant 1$ using an Euler's backwards scheme

$$
\begin{cases}\frac{1}{\Delta t}\left(\theta_{i}^{n}-\theta_{i}^{n-1}\right)=-\left(C \Pi \partial_{a} \widehat{u}^{n-1}, \eta_{i}\right)_{Y}+k\left(C \Pi \partial_{x x} \widehat{u}^{n-1}, \eta_{i}\right)_{Y} & \\ \quad-\sigma \theta_{i}^{n}+\sigma\left(y^{n}, \eta_{i}\right)_{Y}, & 1 \leqslant i \leqslant N, \\ \frac{1}{\Delta t}\left(\widehat{u}^{n}(a, x)-\widehat{u}^{n-1}(a, x)\right)+\partial_{a} \widehat{u}^{n}(a, x)-k \partial_{x x} \widehat{u}^{n}(a, x) & \\ \quad+\sigma \sum_{i=1}^{N} \theta_{i}^{n} v_{i}(a, x)=\sigma \sum_{i=1}^{N}\left(y^{n}, \eta_{i}\right)_{Y} v_{i}(a, x), & a \in\left(0, a^{*}\right), x \in(0, \ell), \\ \theta_{i}^{0}=0, & 1 \leqslant i \leqslant N, \\ \widehat{u}^{n}(a, 0)=\widehat{u}^{n}(a, \ell)=0, & a \in\left(0, a^{*}\right), \\ \widehat{u}^{0}(a, x)=0, & a \in\left(0, a^{*}\right), x \in(0, \ell), \\ \widehat{u}^{n}(0, x)=\int_{0}^{a^{*}} m(a) \widehat{u}^{n-1}(a, x) \mathrm{d} a, & x \in(0, \ell) .\end{cases}
$$

\subsubsection{Finite difference discretization in space}

Denoting by $\widehat{u}_{i}^{n}(a)$ an approximation of $\widehat{u}^{n}\left(x_{i}, a\right)$ (where $x_{i}=i h=i \ell /\left(N_{x}+1\right)$, with $0 \leqslant i \leqslant N_{x}+1$ ) and using a classical centered approximation for the second order derivative in space, the above system yields

$$
\left\{\begin{aligned}
\frac{1}{\Delta t} \theta_{i}^{n}= & \frac{1}{\Delta t} \theta_{i}^{n-1}-\sigma \theta_{i}^{n}-h\left(\int_{a_{1}}^{a_{2}} \Pi(a) \boldsymbol{\eta}_{i}(a)^{\mathbf{T}} \partial_{a} \widehat{\mathbf{U}}^{n-1}(a) \mathrm{d} a\right) \\
& -\frac{1}{h}\left(\int_{a_{1}}^{a_{2}} \Pi(a) \boldsymbol{\eta}_{i}(a)^{\mathbf{T}} \mathbb{K}_{\mathbf{U}^{n-1}}(a) \mathrm{d} a\right)+\sigma h\left(\int_{a_{1}}^{a_{2}} \boldsymbol{\eta}_{i}(a)^{\mathbf{T}} \mathbf{Y}^{n}(a) \mathrm{d} a\right) \\
\frac{\mathrm{d} \widehat{\mathbf{U}}^{n}}{\mathrm{~d} a}(a) & +\frac{1}{h^{2}} \mathbb{K}^{\mathbf{U}^{n}}(a)+\frac{1}{\Delta t} \widehat{\mathbf{U}}^{n}(a)+\sigma \sum_{i=1}^{N} \theta_{i}^{n} \mathbf{V}_{i}(a)=\frac{1}{\Delta t} \widehat{\mathbf{U}}^{n-1}(a) \\
& +\sigma h \sum_{i=1}^{N}\left(\int_{a_{1}}^{a_{2}} \boldsymbol{\eta}_{i}(a)^{\mathbf{T}} \mathbf{Y}^{n}(a) \mathrm{d} a\right) \mathbf{V}_{i}(a), \\
\theta_{i}^{0}=0, & \\
\widehat{\mathbf{U}}^{n}(0)= & \int_{0}^{a^{*}} m(a) \widehat{\mathbf{U}}^{n-1}(a) \mathrm{d} a \\
\widehat{\mathbf{U}}^{0}(a)= & 0,
\end{aligned}\right.
$$

where

$$
\begin{gathered}
\widehat{\mathbf{U}}^{n}(a):=\left(\widehat{u}_{1}^{n}(a), \ldots, \widehat{u}_{N_{x}}^{n}(a)\right)^{\mathbf{T}} \quad \mathbf{V}_{i}(a):=\left(v_{i}\left(a, x_{1}\right), \ldots, v_{i}\left(a, x_{N_{x}}\right)\right)^{\mathbf{T}}, \\
\boldsymbol{\eta}_{i}(a):=\left(\eta_{i}\left(a, x_{1}\right), \ldots, \eta_{i}\left(a, x_{N_{x}}\right)\right)^{\mathbf{T}}, \quad \mathbf{Y}^{n}(a):=\left(y^{n}\left(a, x_{1}\right), \ldots, y^{n}\left(a, x_{N_{x}}\right)\right)^{\mathbf{T}} .
\end{gathered}
$$

We take here $y^{n}\left(a, x_{i}\right)=0$ for all $i \in\left\{1, \ldots, N_{x}\right\}$ such that $x_{i} \notin\left(\ell_{1}, \ell_{2}\right)$, for all $a \in\left(0, a^{*}\right)$ and all $n \geqslant 1$.

\subsubsection{Finite difference discretization in age}

We use a Crank-Nicholson scheme to approximate the solution of problem (5.5). Denoting by $\widehat{u}_{i}^{n, k}$ an approximation of $\widehat{u}_{i}^{n}\left(a^{k}\right)$, where $a^{k}=k \Delta a, 0 \leqslant k \leqslant N_{a}, \Delta a=a^{*} / N_{a}, N_{a_{1}}=\left\lfloor a_{1} / \Delta a\right\rfloor, N_{a_{2}}=\left\lceil a_{2} / \Delta a\right\rceil$ and by 
$\widehat{\mathbf{U}}^{n, k}:=\left(\widehat{u}_{1}^{n, k}, \ldots, \widehat{u}_{N_{x}}^{n, k}\right)^{\mathbf{T}}$ an approximation of $\widehat{\mathbf{U}}^{n}\left(a^{k}\right)$, we move from age $a^{k-1}$ to age $a^{k}$ following the scheme

$$
\begin{aligned}
\frac{1}{\Delta t} \theta_{i}^{n}= & \frac{1}{\Delta t} \theta_{i}^{n-1}-\sigma \theta_{i}^{n}-h \Delta a\left(\sum_{k=N_{a_{1}}}^{N_{a_{2}}} \Pi(k \Delta a)\left(\boldsymbol{\eta}_{i}^{k}\right)^{\mathbf{T}}\left(\frac{\widehat{\mathbf{U}}^{n-1, k}-\widehat{\mathbf{U}}^{n-1, k-1}}{\Delta a}\right)\right) \\
& -\frac{\Delta a}{h}\left(\sum_{k=N_{a_{1}}}^{N_{a_{2}}} \Pi(k \Delta a)\left(\boldsymbol{\eta}_{i}^{k}\right)^{\mathbf{T}} \mathbb{K}^{n-1, k}\right)+\sigma h \Delta a\left(\sum_{k=N_{a_{1}}}^{N_{a_{2}}}\left(\boldsymbol{\eta}_{i}^{k}\right)^{\mathbf{T}} \mathbf{Y}^{n, k}\right),
\end{aligned}
$$

and

$$
\begin{aligned}
\frac{1}{\Delta a}\left(\widehat{\mathbf{U}}^{n, k}-\widehat{\mathbf{U}}^{n, k-1}\right)+\frac{1}{2 h^{2}} \mathbb{K}\left(\widehat{\mathbf{U}}^{n, k}\right. & \left.+\widehat{\mathbf{U}}^{n, k-1}\right)+\frac{1}{2 \Delta t}\left(\widehat{\mathbf{U}}^{n, k}+\widehat{\mathbf{U}}^{n, k-1}\right)+\sigma \sum_{i=1}^{N} \theta_{i}^{n} \mathbf{V}_{i}^{k} \\
& =\frac{1}{2 \Delta t}\left(\widehat{\mathbf{U}}^{n-1, k}+\widehat{\mathbf{U}}^{n-1, k-1}\right)+\sigma h \Delta a \sum_{i=1}^{N}\left(\sum_{j=N_{a_{1}}}^{N_{a_{2}}}\left(\boldsymbol{\eta}_{i}^{j}\right)^{\mathbf{T}} \mathbf{Y}^{n, j}\right) \mathbf{V}_{i}^{k},
\end{aligned}
$$

with the initial conditions

$$
\left\{\begin{array}{l}
\theta_{i}^{0}=0, \quad \forall i=1, \ldots, N, \\
\widehat{\mathbf{U}}^{0, k}=0, \quad \forall k=0, \ldots, N_{a}, \\
\widehat{\mathbf{U}}^{n, 0}=\sum_{k=0}^{N_{a}} \omega_{k} m\left(a^{k}\right) \widehat{\mathbf{U}}^{n-1, k} .
\end{array}\right.
$$

Here: $\mathbf{V}_{i}^{k}:=\left(v_{i}\left(k \Delta a, x_{1}\right), \ldots, v_{i}\left(k \Delta a, x_{N_{x}}\right)^{\mathbf{T}}, \quad \boldsymbol{\eta}_{i}^{k}:=\left(\eta_{i}\left(k \Delta a, x_{1}\right), \ldots, \eta_{i}\left(k \Delta a, x_{N_{x}}\right)\right)^{\mathbf{T}}\right.$, and $\quad \mathbf{Y}^{n, k}:=$ $\left.\left(y^{n}\left(k \Delta a, x_{1}\right), c y^{n}\left(k \Delta a, x_{N_{x}}\right)\right)\right)^{\mathbf{T}}$.

\subsubsection{Algorithm for the observer}

(1) For $n=0$ : Initialization of $\theta_{i}^{0}(1 \leqslant i \leqslant N)$ and $\widehat{\mathbf{U}}^{0, k}\left(k=0, \ldots, N_{a}\right)$ at 0 .

(2) For $n=1, \ldots, N_{t}$ :

- Calculate $\theta_{i}^{n}$ using the values of $\theta_{i}^{n-1}$ and $\left(\widehat{\mathbf{U}}^{n-1, j}\right)_{j=N_{a_{1}-1}}^{N_{a_{2}}}$ :

$$
\begin{array}{r}
\theta_{i}^{n}=\frac{1}{1+\sigma \Delta t}\left(\theta_{i}^{n-1}-h \Delta t \sum_{k=N_{a_{1}}}^{N_{a_{2}}} \Pi(k \Delta a)\left(\boldsymbol{\eta}_{i}^{k}\right)^{\mathbf{T}}\left(\widehat{\mathbf{U}}^{n-1, k}-\widehat{\mathbf{U}}^{n-1, k-1}\right)\right. \\
\left.\quad-\frac{\Delta a \Delta t}{h} \sum_{k=N_{a_{1}}}^{N_{a_{2}}} \Pi(k \Delta a)\left(\boldsymbol{\eta}_{i}^{k}\right)^{\mathbf{T}} \mathbb{K}^{n-1, k}+\sigma h \Delta a \Delta t \sum_{k=N_{a_{1}}}^{N_{a_{2}}}\left(\boldsymbol{\eta}_{i}^{k}\right)^{\mathbf{T}} \mathbf{Y}^{n, k}\right)
\end{array}
$$

- $k=0$ : Initialization of $\widehat{\mathbf{U}}^{n, 0}$ using the values of $\left(\widehat{\mathbf{U}}^{n-1, j}\right)_{j=0}^{N_{a}}$ :

$$
\widehat{\mathbf{U}}^{n, 0}=\sum_{k=0}^{N_{a}} \omega_{k} m(k \Delta a) \widehat{\mathbf{U}}^{n-1, k}
$$

- For $k=1, \ldots, N_{a}$, solve the linear system

$$
\mathbb{A} \widehat{\mathbf{U}}^{n, k}=\widehat{\mathbf{b}}^{n, k}
$$


Time $: n=0, \ldots, N_{t}$

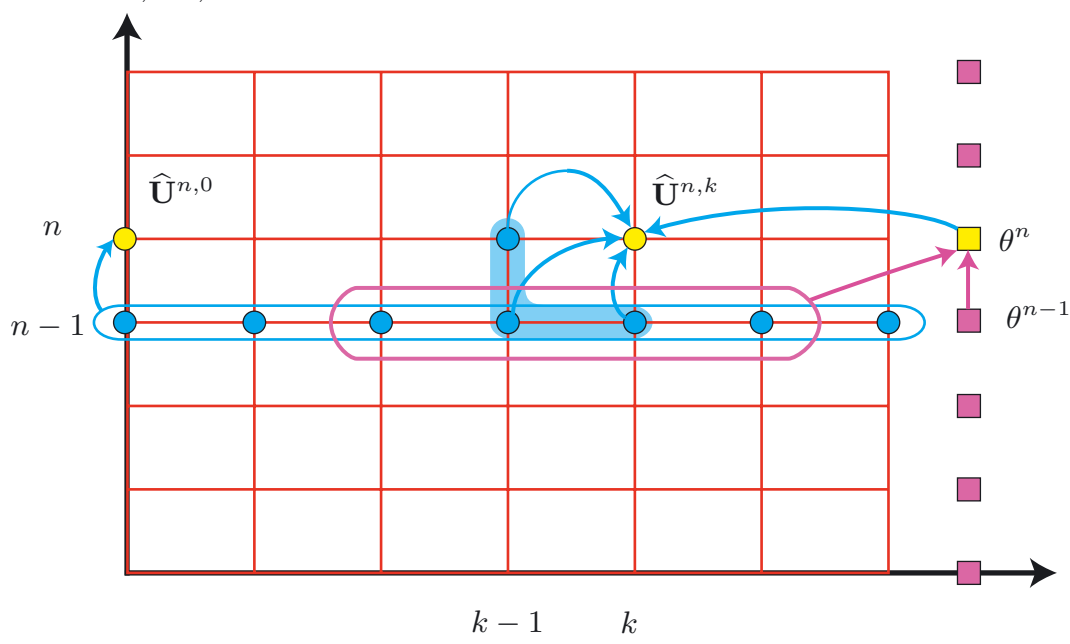

Age $: k=0, \ldots, N_{a}$

FiguRE 4. Step $n$ of the algorithm. First, $\theta_{i}^{n}$ is computed using $\theta_{i}^{n-1}$ and $\widehat{\mathbf{U}}^{n-1, k}$, for $k=$ $N_{a_{1}}-1, \ldots, N_{a_{2}}$ (pink arrows). Next, $\widehat{\mathbf{U}}^{n, 0}$ is deduced from $\widehat{\mathbf{U}}^{n-1, k}$, for $k=0, \ldots, N_{a}$. Finally, $\widehat{\mathbf{U}}^{n, k}$ for $k \geqslant 1$ is computed using $\theta_{i}^{n}, \widehat{\mathbf{U}}^{n, k-1}, \widehat{\mathbf{U}}^{n-1, k}$ and $\widehat{\mathbf{U}}^{n-1, k-1}$ (blue arrows). (Color online)

where

$$
\begin{gathered}
\mathbb{A}=\left(\Delta t+\frac{1}{2} \Delta a\right) \mathbb{I}+\frac{1}{2} \frac{\Delta t \Delta a}{h^{2}} \mathbb{K} \\
\widehat{\mathbf{b}}^{n, k}=\frac{1}{2} \Delta a\left(\widehat{\mathbf{U}}^{n-1, k}+\widehat{\mathbf{U}}^{n-1, k-1}\right)+\left[\left(\Delta t-\frac{1}{2} \Delta a\right) \mathbb{I}-\frac{1}{2} \frac{\Delta t \Delta a}{h^{2}} \mathbb{K}\right] \widehat{\mathbf{U}}^{n, k-1} \\
-\sigma \Delta a \Delta t \sum_{i=1}^{N} \theta_{i}^{n} \mathbf{V}_{i}^{k}+\sigma h(\Delta a)^{2} \Delta t \sum_{i=1}^{N}\left(\sum_{j=N_{a_{1}}}^{N_{a_{2}}}\left(\boldsymbol{\eta}_{i}^{j}\right)^{\mathbf{T}} \mathbf{Y}^{n, j}\right) \mathbf{V}_{i}^{k}
\end{gathered}
$$

- $\widehat{\mathbf{P}^{n, k}}=\left(\widehat{p}_{1}^{n, k}, \ldots, \widehat{p}_{N_{x}}^{n, k}\right)^{\mathbf{T}}$ where $\widehat{p}_{i}^{n, k}=\Pi\left(a^{k}\right) \widehat{u}_{i}^{n, k}$.

\section{Numerical RESUlts}

We present in this section some numerical results illustrating the efficiency of our state reconstruction method. All the numerical tests are two-dimensional and run on Matlab.

Taking $a^{*}=2$, we choose the fertility function to be (see Fig. 5)

$$
\beta(a)=10 a\left(a^{*}-a\right) \exp \left\{-20\left(a-a^{*} / 3\right)^{2}\right\},
$$

while the mortality function is chosen as

$$
\mu(a)=\left(a^{*}-a\right)^{-1} .
$$

Note that for this particular choice of $\mu$, the function $\Pi(a)$ can be computed explicitly:

$$
\Pi(a)=\exp \left(-\int_{0}^{a} \mu(s) \mathrm{d} s\right)=\frac{a^{*}-a}{a^{*}} .
$$




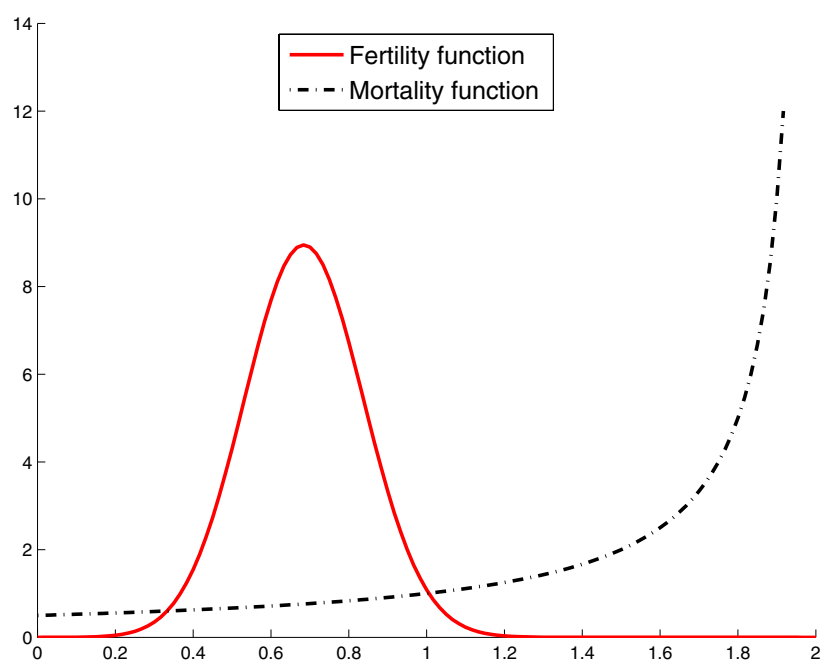

FiguRE 5. The fertility and mortality functions.
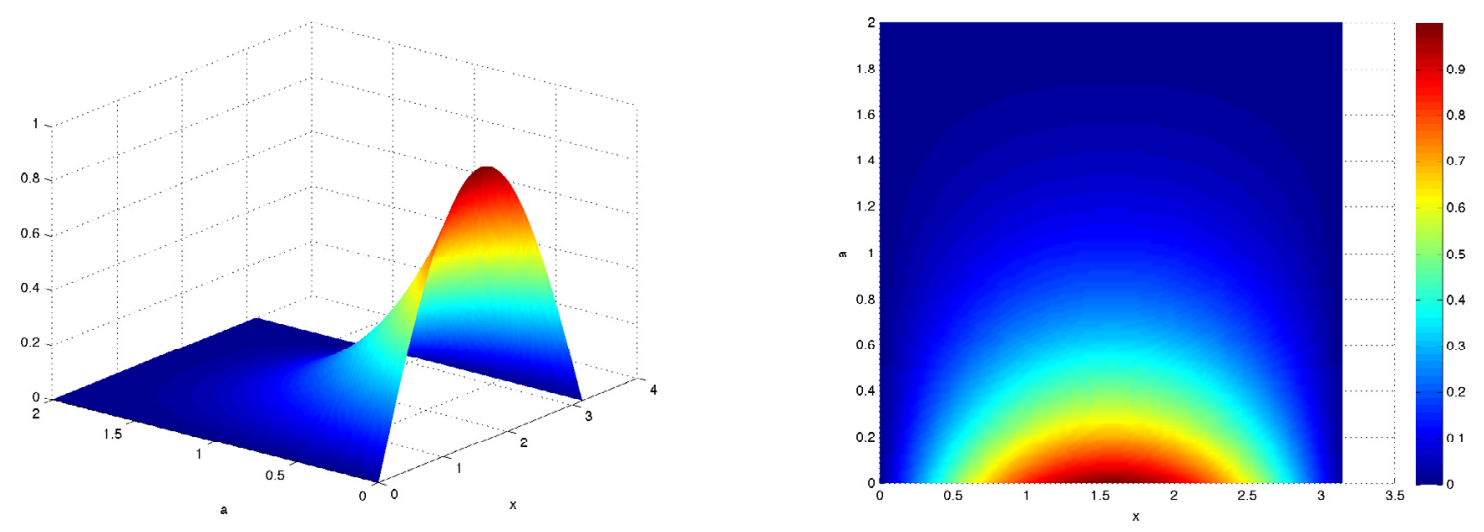

FIGURE 6. Initial state corresponding to the first eigenfunction (3D and $2 \mathrm{D}$ representations).

\subsection{Distributed observation in space and full observation in age}

We take $\Omega=(0, \pi)$ (i.e. $\ell=\pi)$, and we consider full observation in age (i.e. $\left(a_{1}, a_{2}\right)=\left(0, a^{*}\right)$ ) and partial observation in space $:\left(\ell_{1}, \ell_{2}\right)=(\ell / 3,2 \ell / 3)$. The time of observation is $T=2 a^{*}$ and the diffusion coefficient is $k=1$. Under these assumptions, there is a unique unstable eigenvalue $\lambda_{1}=\lambda_{1}^{0}-1$, where $\lambda_{1}^{0}$ is the unique real solution of the characteristic equation (2.2). Computing numerically this value, we obtain that $\lambda_{1}=0.239$.

We first choose as initial state an eigenfunction corresponding to the (unique) unstable eigenvalue $\lambda_{1}$ (see Fig. 6), i.e. we take

$$
p_{0}(a, x)=\varphi_{1}(a, x)=\varphi_{1}^{0}(a) \varphi_{1}^{D}(x)=\frac{a^{*}-a}{a^{*}} \mathrm{e}^{-\lambda_{1}^{0} a} \sin (x) .
$$



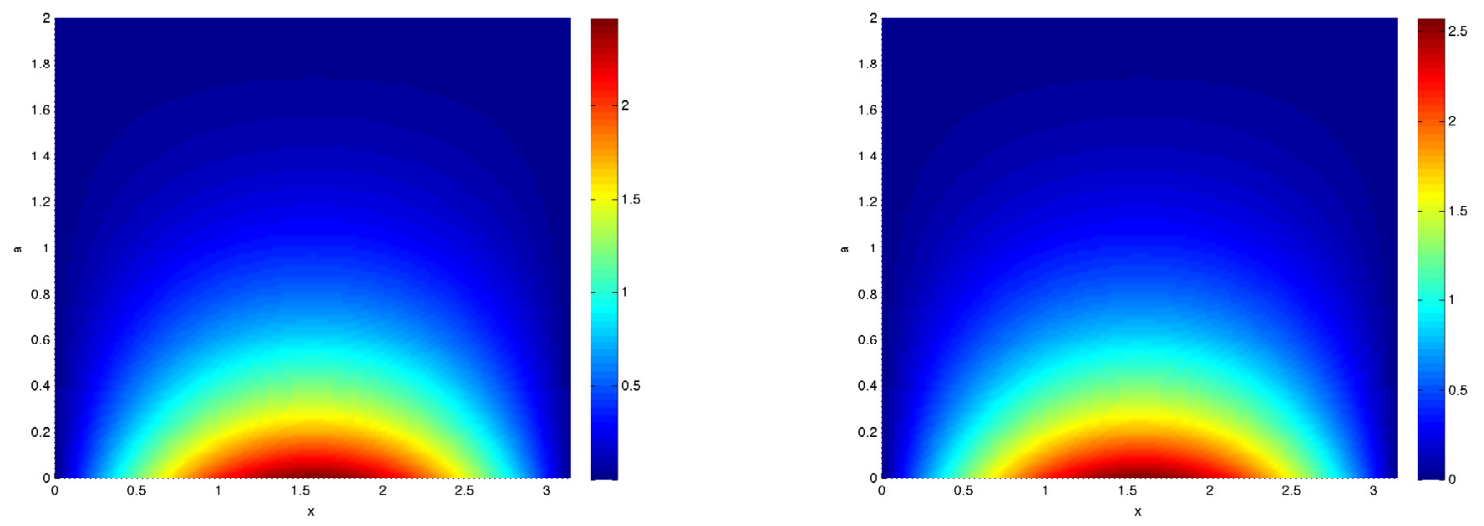

Figure 7. Estimated (left) and exact (right) solution at time $t=T$.
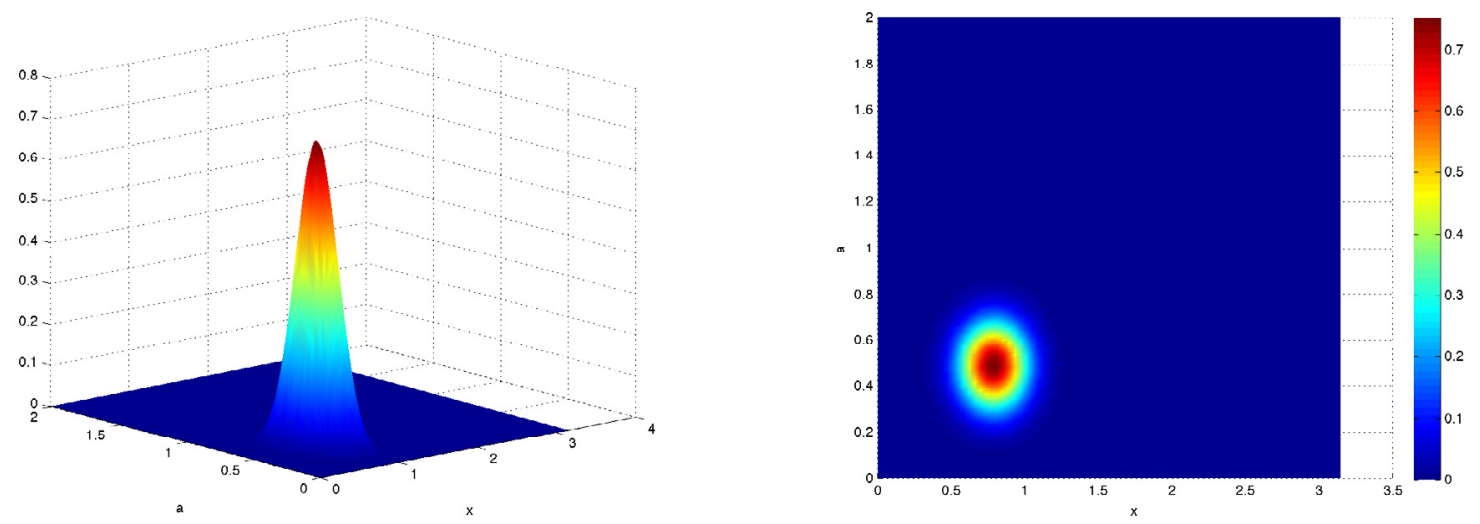

FiguRE 8. Gaussian initial state (3D and 2D representations).

The solution of problem (5.1) is then simply given by

$$
p(a, x, t)=\mathrm{e}^{\lambda_{1} t} p_{0}(a, x) .
$$

To validate our reconstruction method, we first generate numerically an output by solving problem (5.1) with $p_{0}$ given by (6.1). To avoid committing an "inverse crime" ([16], p. 154), we use different space discretizations to generate the measurement and in the reconstruction step.

Using $N_{x}=100$ points of discretization in space, $N_{a}=120$ points in discretization in age and a time step $\Delta t=\Delta a / 2$, we obtain an $L^{2}$ relative error of $4.07 \%$ (see Fig. 7 for a representation of the estimated and exact solutions). In this numerical test, the gain coefficient is chosen as $\sigma=2+\lambda_{1}$.

From now on, we consider the more realistic situation of noisy observation and gaussian type initial data of the form (see Fig. 8)

$$
p_{0}(a, x)=\exp \left\{-\left(30\left(a-a^{*} / 4\right)^{2}+20(x-\ell / 4)^{2}\right)\right\} .
$$

Using the same discretization parameters, we obtain an $L^{2}$ relative error of $2.99 \%$ with $5 \%$ of noise, $9.6 \%$ with $10 \%$ of noise and $16.3 \%$ with $15 \%$ of noise; see Figure 9 for a representation of the estimated and exact solutions at time $t=T$ in the case of $5 \%$ of noise. Figure 10 shows the decay of the state error with time. 

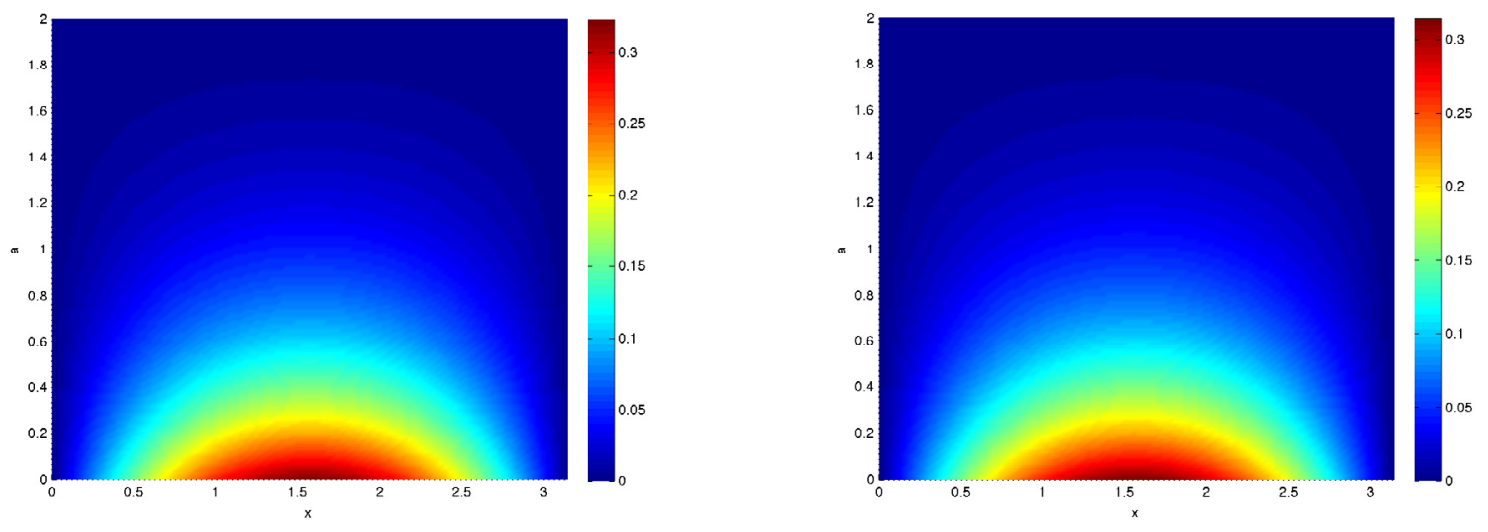

Figure 9. Estimated (left) and exact (right) solution at time $t=T$ (with $5 \%$ of noise).

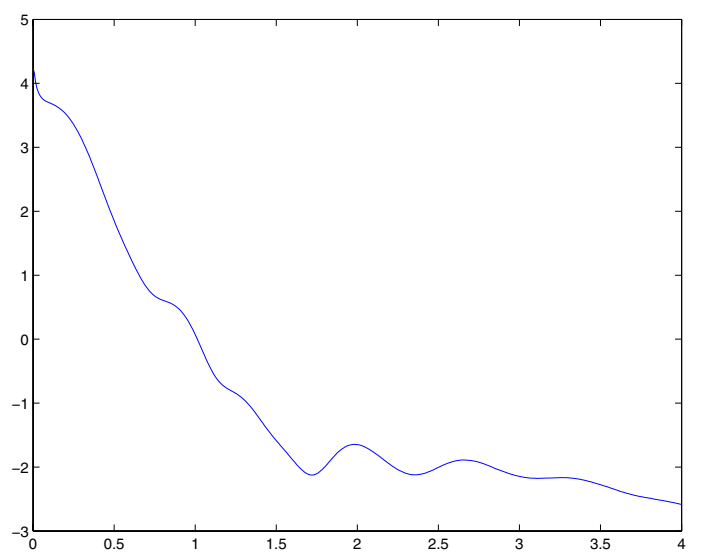

Figure 10. Decay of the relative error (in log scale) with respect to time (with $5 \%$ of noise).

We also plot on Figure 11 the estimated and exact ${ }^{3}$ final total population : $P_{T}(x)=\int_{0}^{a^{*}} p(a, T, x) \mathrm{d} a$ and $\widehat{P}_{T}(x)=\int_{0}^{a^{*}} \widehat{p}(a, T, x) \mathrm{d} a$.

Remark 6.1. One might wonder why the fact that the estimated final states obtained for two different initial data (the first eigenfunction and an initial state of gaussian type) look the same. In fact, this is not surprising as the asymptotic behavior (in time) of the solution of (1.1) is given by the first eigenfunction when there is only one unstable eigenvalue.

Surprisingly enough, the reconstruction method is efficient even when the space interval of observation $\left(\ell_{1}, \ell_{2}\right)$ is small. More precisely, for $N_{x}=60, N_{a}=80$ and $10 \%$ of noise, we obtain an $L^{2}$-relative error of $19.9 \%$ when $\ell_{2}-\ell_{1}=3 h, h$ being the mesh size (in space). The same experiment run for $\ell_{2}-\ell_{1}=\ell / 3$ leads to a relative error of $9.1 \%$.

\footnotetext{
${ }^{3}$ Here, what we refer to as the "exact" solution is a numerical solution, computed assuming the initial data to be known.
} 

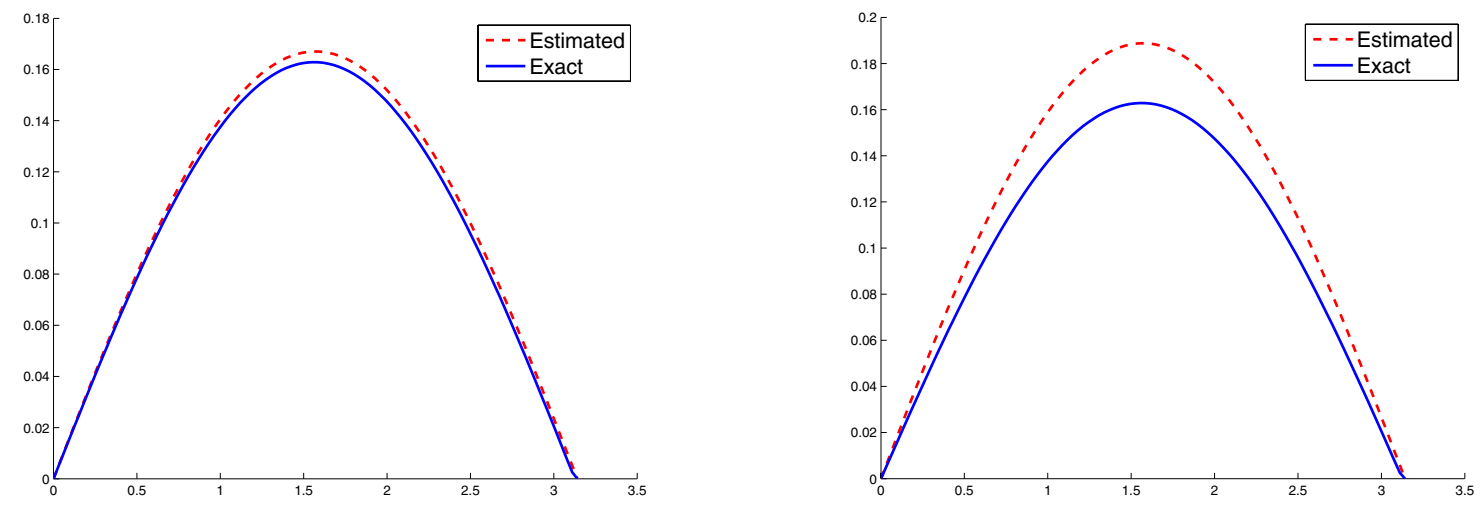

FiguRE 11. Estimated (dashed line) and exact total population at time $t=T$ with $5 \%$ of noise (left) and $15 \%$ of noise (right).
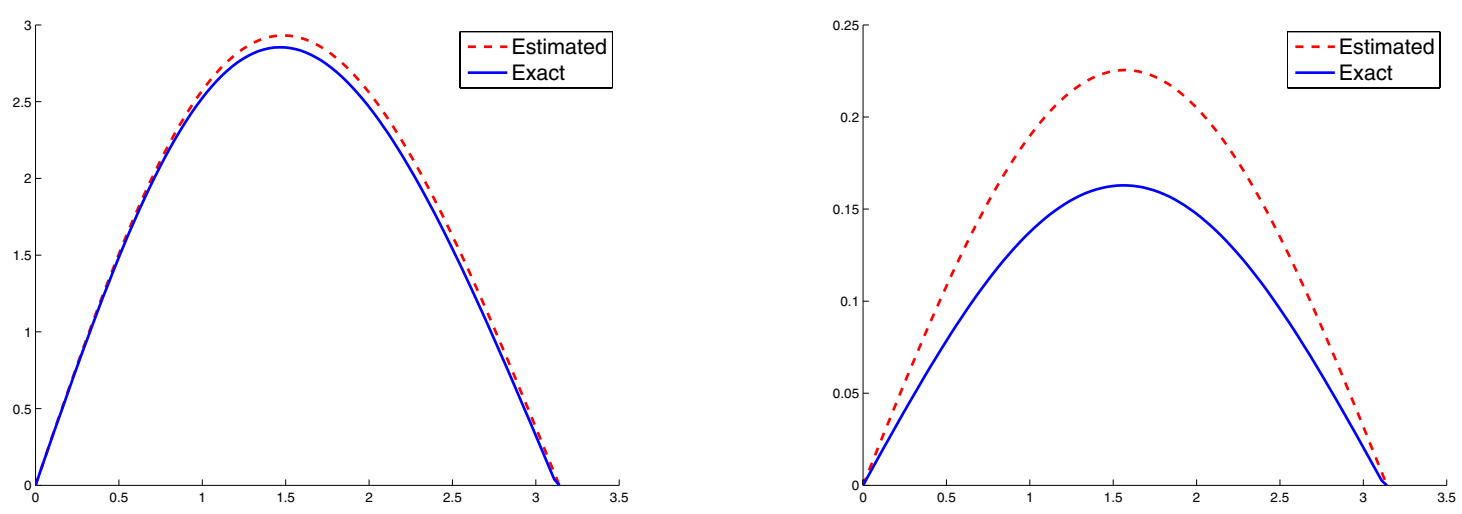

Figure 12. Estimated (dashed line) and exact total population at time $t=T$ with age observation near $a=0$ (left) and far from $a=0$ (right).

\subsection{Distributed observation in space and age}

We consider now the case of local observation in age by taking successively $\left(a_{1}, a_{2}\right)=\left(0, a^{*} / 40\right)$ and $\left(a_{1}, a_{2}\right)=$ $\left(a^{*} / 2, a^{*}\right)$. The observation interval in space is chosen in both cases to be $(\ell / 3,2 \ell / 3)$ and we use noisy data with $5 \%$ of noise. The relative errors on the final state are then respectively of $3.3 \%$ and $38.8 \%$ (see Fig. 12). This shows that the quality of the reconstruction is not influenced by the size of the age observation interval but mainly by its location. One can obtain a very good reconstruction provided this interval is located near the origin, even for a pointwise observation (see Rem. 4.6). This shows the crucial role played by the observation of births in the quality of the observer.

\subsection{Influence of the observation time}

In this last experiment, we consider a configuration where there are two unstable eigenvalues $\left(\lambda_{1}=0.959\right.$ and $\left.\lambda_{2}=0.119\right)$. This can be done by reducing the value of the diffusion coefficient $k=0.28$, and taking the 

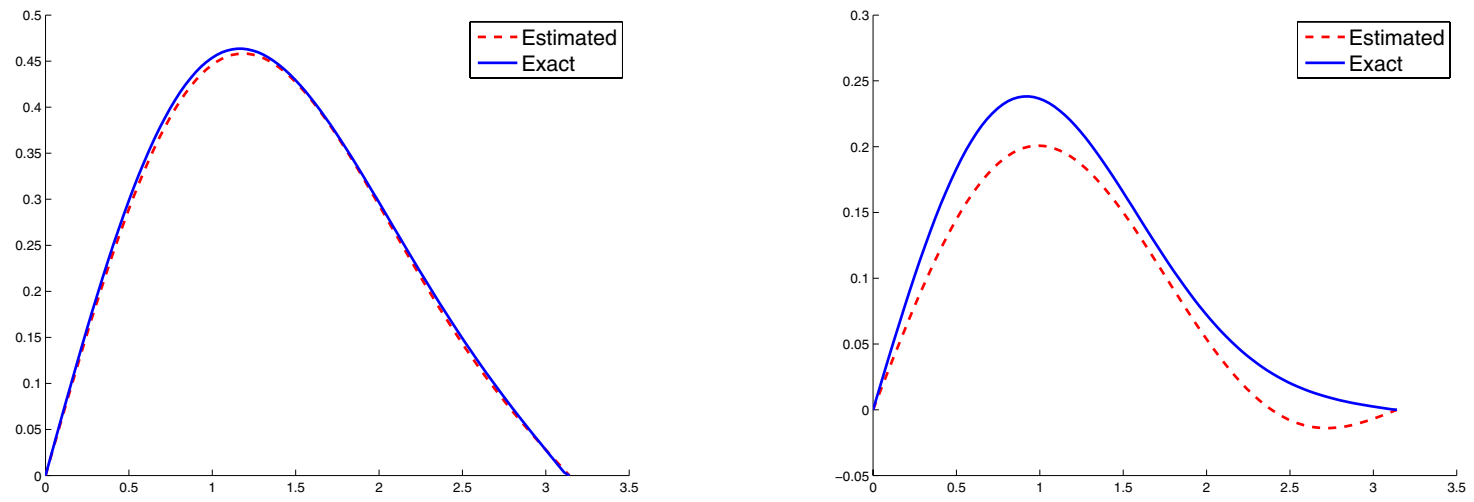

Figure 13. Estimated (dashed line) and exact total population at time $t=T$, for $T=a^{*}$ (left) and for $T=0.5 a^{*}$ (right).

same parameters as in the previous numerical test. The observation interval in space is chosen to be $(\ell / 3,2 \ell / 3)$ and $\left(a_{1}, a_{2}\right)=\left(0, a^{*} / 20\right)$.

For $T=a^{*}$, the observer yields a good approximation of the final state (the relative error is $3.83 \%$ ), and an even better approximation of the total population (the relative error is $1.81 \%$ ). For an observation time $T=0.5 a^{*}$, the estimate provided by the observer is not very accurate, the relative error being $27 \%$. However, we still obtain a reasonable approximation of the total population, as it can be seen from Figure 13.

\section{REFERENCES}

[1] B. Ainseba, Exact and approximate controllability of the age and space population dynamics structured model. J. Math. Anal. Appl. 275 (2002) 562-574.

[2] B. Ainseba and M. Iannelli, Exact controllability of a nonlinear population-dynamics problem. Differ. Integral Equ. 16 (2003) $1369-1384$.

[3] B. Ainseba and M. Langlais, On a population dynamics control problem with age dependence and spatial structure. J. Math. Anal. Appl. 248 (2000) 455-474.

[4] D. Auroux and J. Blum, Back and forth nudging algorithm for data assimilation problems. C. R. Acad. Sci. Paris Sér. I Math. 340 (2005) 873-878.

[5] D. Auroux and M. Nodet, The back and forth nudging algorithm for data assimilation problems: theoretical results on transport equations. ESAIM: COCV 18 (2012) 318-342.

[6] D. Auroux, P. Bansart and J. Blum, An evolution of the back and forth nudging for geophysical data assimilation: application to Burgers equation and comparisons. Inverse Probl. Sci. Eng. 21 (2013) 399-419.

[7] B.P. Ayati, A variable time step method for an age-dependent population model with nonlinear diffusion. SIAM J. Numer. Anal. 37 (2000) 1571-1589.

[8] B.P. Ayati and T.F. Dupont, Galerkin methods in age and space for a population model with nonlinear diffusion. SIAM J. Numer. Anal. 40 (2002) 1064-1076.

[9] M. Badra and T. Takahashi, Stabilization of parabolic nonlinear systems with finite dimensional feedback or dynamical controllers: application to the Navier-Stokes system. SIAM J. Control Optim. 49 (2011) 420-463.

[10] M. Badra and T. Takahashi, On the Fattorini criterion for approximate controllability and stabilizability of parabolic systems. ESAIM: COCV 20 (2014) 924-956.

[11] J.S. Baras, A. Bensoussan and M.R. James, Dynamic observers as asymptotic limits of recursive filters: special cases. SIAM J. Appl. Math. 48 (1988) 1147-1158.

[12] V. Barbu and R. Triggiani, Internal stabilization of Navier-Stokes equations with finite-dimensional controllers. Indiana Univ. Math. J. 53 (2004) 1443-1494.

[13] W.L. Chan and B.Z. Guo, On the semigroups of age-size dependent population dynamics with spatial diffusion. Manuscr. Math. 66 (1989) 161-181.

[14] D. Chapelle, N. Cîndea and P. Moireau, Improving convergence in numerical analysis using observers-the wave-like equation case. Math. Models Methods Appl. Sci. 22 (2012) 1250040. 
[15] M.J. Chapman and A.J. Pritchard, Finite-dimensional compensators for nonlinear infinite-dimensional systems, in Control theory for distributed parameter systems and applications (Vorau, 1982). Vol. 54 of Lect. Notes Control Inform. Sci. Springer, Berlin (1983) 60-76.

[16] D. Colton and R. Kress, Inverse acoustic and electromagnetic scattering theory. Vol. 93 of Appl. Math. Sci., 2nd edition. Springer-Verlag, Berlin, (1998).

[17] J.-M. Coron and E. Trélat, Global steady-state controllability of one-dimensional semilinear heat equations. SIAM J. Control Optim. 43 (2004) 549-569.

[18] R.F. Curtain and H. Zwart, An introduction to infinite-dimensional linear systems theory. Vol. 21 of Texts Appl. Math. Springer-Verlag, New York (1995).

[19] R. Curtain, M. Demetriou and K. Ito, Adaptive observers for structurally perturbed infinite dimensional systems, in Proc. of the 36th IEEE Conference on Decision and Control, 1997, Vol. 1 (1997) 509-514.

[20] C. Cusulin and L. Gerardo-Giorda, A numerical method for spatial diffusion in age-structured populations. Numer. Methods Partial Differ. Equ. 26 (2010) 253-273.

[21] G. Di Blasio and A. Lorenzi, Direct and inverse problems in age-structured population diffusion. Discrete Contin. Dyn. Syst. Ser. $S 4$ (2011) 539-563.

[22] G. Di Blasio and A. Lorenzi, An identification problem in age-dependent population diffusion. Numer. Funct. Anal. Optim. 34 (2013) 36-73.

[23] H.W. Engl, W. Rundell and O. Scherzer, A regularization scheme for an inverse problem in age-structured populations. J. Math. Anal. Appl. 182 (1994) 658-679.

[24] E. Fridman, Observers and initial state recovering for a class of hyperbolic systems via Lyapunov method. Automatica 49 (2013) 2250-2260.

[25] L. Gerardo-Giorda, Numerical approximation of density dependent diffusion in age-structured population dynamics, in Conference Applications of Mathematics 2013 in honor of the 70th birthday of Karel Segeth, edited by J. Brandts, S. Korotov, M. Kryzek, J. Sistek and T. Vejchodsky. Vol. 2013. Institute of Mathematics AS CR, Prague (2013) 88-97.

[26] B.Z. Guo and W.L. Chan, On the semigroup for age dependent population dynamics with spatial diffusion. J. Math. Anal. Appl. 184 (1994) 190-199.

[27] M.E. Gurtin, A system of equations for age-dependent population diffusion. J. Theoret. Biol. 40 (1973).

[28] M.E. Gurtin and R.C. MacCamy, Diffusion models for age-structured populations. Math. Biosci. 54 (1981) $49-59$.

[29] M. Gyllenberg, A. Osipov and L. Päivärinta, The inverse problem of linear age-structured population dynamics. J. Evol. Equ. 2 (2002) 223-239.

[30] G. Haine, Recovering the observable part of the initial data of an infinite-dimensional linear system with skew-adjoint generator. Math. Control. Signals Syst. 26 (2014) 435-462.

[31] G. Haine and K. Ramdani, Reconstructing initial data using observers: error analysis of the semi-discrete and fully discrete approximations. Numer. Math. 120 (2012) 307-343.

[32] Z. Hidayat, R. Babuska, B. De Schutter and A. Nunez, Observers for linear distributed-parameter systems: A survey, in IEEE International Symposium on, Robotic and Sensors Environments (ROSE) (2011) 166-171.

[33] W. Huyer, Semigroup formulation and approximation of a linear age-dependent population problem with spatial diffusion. Semigroup Forum 49 (1994) 99-114.

[34] W. Huyer, Approximation of a linear age-dependent population model with spatial diffusion. Commun. Appl. Anal. 8 (2004) $87-108$.

[35] M.R. James and J.S. Baras, An observer design for nonlinear control systems, in Analysis and optimization of systems (Antibes, 1988). Vol. 111 of Lect. Notes Control Inform. Sci. Springer, Berlin (1988) 170-180.

[36] T. Kato, Perturbation theory for linear operators. Classics in Mathematics. Springer-Verlag, Berlin (1995).

[37] O. Kavian and O. Traoré, Approximate controllability by birth control for a nonlinear population dynamics model. ESAIM: COCV 17 (2011) 1198-1213.

[38] M.-Y. Kim, Galerkin methods for a model of population dynamics with nonlinear diffusion. Numer. Methods Partial Differ. Equ. 12 (1996) 59-73.

[39] M.-Y. Kim and E.-J. Park, Mixed approximation of a population diffusion equation. Comput. Math. Appl. 30 (1995) 23-33.

[40] M. Krstic, L. Magnis and R. Vazquez, Nonlinear control of the viscous Burgers equation: Trajectory generation, tracking and observer design. J. Dyn. Syst. Meas. Control 131 (2009) 021012.

[41] M. Langlais, A nonlinear problem in age-dependent population diffusion. SIAM J. Math. Anal. 16 (1985) $510-529$.

[42] M. Langlais, Large time behavior in a nonlinear age-dependent population dynamics problem with spatial diffusion $J$. Math. Biol. 26 (1988) 319-346.

[43] L. Lopez and D. Trigiante, A finite difference scheme for a stiff problem arising in the numerical solution of a population dynamic model with spatial diffusion. Nonlin. Anal. 9 (1985) 1-12.

[44] D. Luenberger, Observing the state of a linear system, IEEE Trans. Mil. Electron. MIL-8 (1964) 74-80.

[45] F.A. Milner, A numerical method for a model of population dynamics with spatial diffusion. Comput. Math. Appl. 19 (1990) 31-43.

[46] P. Moireau, D. Chapelle and P. Le Tallec, Joint state and parameter estimation for distributed mechanical systems. Comput. Methods Appl. Mech. Eng. 197 (2008) 659-677.

[47] P. Moireau, D. Chapelle and P. Le Tallec, Filtering for distributed mechanical systems using position measurements: perspectives in medical imaging Inverse Probl. 25 (2009) 035010. 
[48] G.G. Pelovska, Numerical investigations in the field of age-structured population dynamics. Ph.D. thesis, University of Trento (2007).

[49] A. Perasso, Identifiabilité de paramètres pour des systèmes décrits par des équations aux dérivées partielles. Application à la dynamique des populations. Ph.D. thesis, Université Paris Sud XI (2009).

[50] K.D. Phung and G. Wang, An observability estimate for parabolic equations from a measurable set in time and its applications. J. Eur. Math. Soc. 15 (2013) 681-703.

[51] M. Pilant and W. Rundell, Determining a coefficient in a first-order hyperbolic equation. SIAM J. Appl. Math. 51 (1991) 494-506.

[52] J.-P. Puel, Une approche non classique d'un problème d'assimilation de données. C. R. Math. Acad. Sci. Paris 335 (2002) $161-166$.

[53] J.-P. Puel, A nonstandard approach to a data assimilation problem and Tychonov regularization revisited. SIAM J. Control Optim. 48 (2009) 1089-1111.

[54] K. Ramdani, M. Tucsnak and G. Weiss, Recovering the initial state of an infinite-dimensional system using observers. Automatica 46 (2010) 1616-1625.

[55] J.-P. Raymond and L. Thevenet, Boundary feedback stabilization of the two dimensional Navier-Stokes equations with finite dimensional controllers. Discrete Contin. Dyn. Syst. 27 (2010) 1159-1187.

[56] A. Rhandi, Positivity and stability for a population equation with diffusion on $L^{1}$. Positivity 2 (1998) $101-113$.

[57] W. Rundell, Determining the death rate for an age-structured population from census data. SIAM J. Appl. Math. 53 (1993) $1731-1746$.

[58] A. Smyshlyaev and M. Krstic, Backstepping observers for a class of parabolic PDEs. Syst. Control Lett. 54 (2005) 613-625.

[59] J. Song, J.Y. Yu, X.Z. Wang, S.J. Hu, Z.X. Zhao, J.Q. Liu, D.X. Feng and G.T. Zhu, Spectral properties of population operator and asymptotic behaviour of population semigroup. Acta Math. Sci. 2 (1982) 139-148.

[60] O. Traore, Null controllability of a nonlinear population dynamics problem. Int. J. Math. Math. Sci. 20 (2006) 49279.

[61] O. Traore, Approximate controllability and application to data assimilation problem for a linear population dynamics model. Int. J. Appl. Math. 37 (2007) 1, 12.

[62] O. Traore, Null controllability and application to data assimilation problem for a linear model of population dynamics. Ann. Math. Blaise Pascal 17 (2010) 375-399.

[63] R. Triggiani, On the stabilizability problem in Banach space. J. Math. Anal. Appl. 52 (1975) 383-403.

[64] R. Triggiani, Addendum. J. Math. Anal. Appl. 56 (1976) 492-493.

[65] R. Triggiani, Boundary feedback stabilizability of parabolic equations. Appl. Math. Optim. 6 (1980) 201-220.

[66] C. Walker, Some remarks on the asymptotic behavior of the semigroup associated with age-structured diffusive populations. Monatsh. Math. 170 (2013) 481-501.

[67] G.F. Webb, Theory of nonlinear age-dependent population dynamics. Vol. 89 of Monogr. Textb. Pure Appl. Math. Marcel Dekker, Inc., New York (1985).

[68] G.F. Webb, Population models structured by age, size and spatial position, in Structured population models in biology and epidemiology. Vol. 1936 of Lect. Notes Math. Springer, Berlin (2008) 1-49.

[69] J. Zabczyk, Remarks on the algebraic Riccati equation in Hilbert space. Appl. Math. Optim. 2 (1975/76) 251-258. 